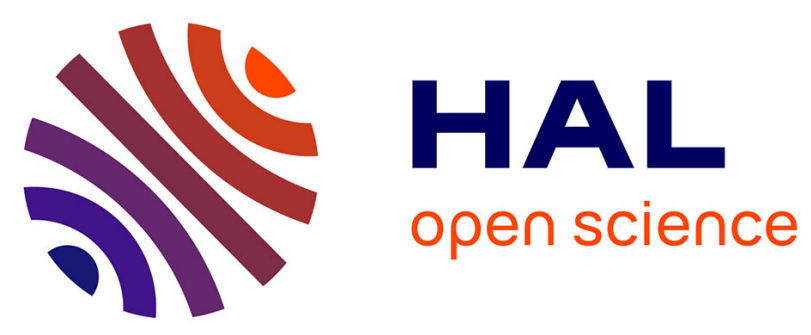

\title{
Understanding the reactivity of unsaturated alcohols: Experimental and kinetic modeling study of the pyrolysis and oxidation of 3-methyl-2-butenol and 3-methyl-3-butenol
}

Ruben de Bruycker, Olivier Herbinet, Hans-Heinrich Carstensen, Frédérique

Battin Leclerc, Kevin M. van Geem

\section{To cite this version:}

Ruben de Bruycker, Olivier Herbinet, Hans-Heinrich Carstensen, Frédérique Battin Leclerc, Kevin M. van Geem. Understanding the reactivity of unsaturated alcohols: Experimental and kinetic modeling study of the pyrolysis and oxidation of 3-methyl-2-butenol and 3-methyl-3-butenol. Combustion and Flame, 2016, 171, pp.237-251. 10.1016/j.combustflame.2016.06.009 . hal-01349463

\author{
HAL Id: hal-01349463 \\ https://hal.science/hal-01349463
}

Submitted on 27 Jul 2016

HAL is a multi-disciplinary open access archive for the deposit and dissemination of scientific research documents, whether they are published or not. The documents may come from teaching and research institutions in France or abroad, or from public or private research centers.
L'archive ouverte pluridisciplinaire HAL, est destinée au dépôt et à la diffusion de documents scientifiques de niveau recherche, publiés ou non, émanant des établissements d'enseignement et de recherche français ou étrangers, des laboratoires publics ou privés. 


\title{
Understanding the reactivity of unsaturated alcohols: Experimental and kinetic modeling study of the pyrolysis and oxidation of 3-methyl-2-butenol and 3-methyl-3-butenol
}

\author{
Ruben De Bruycker ${ }^{1}$, Olivier Herbinet ${ }^{2}$, Hans-Heinrich Carstensen1, Frédérique \\ Battin-Leclerc ${ }^{2}$, Kevin M. Van Geem ${ }^{1, *}$
}

${ }^{1}$ Laboratory for Chemical Technology, Ghent University, Technologiepark 914, 9052 Gent, Belgium ${ }^{2}$ Laboratoire Réactions et Génie des Procédés, CNRS, Université de Lorraine, Nancy, France

Published in Combustion and Flame (2016) 271, 137-151

doi:10.1016/j.combustflame.2016.06.009

\begin{abstract}
The reactivity of unsaturated alcohols with a $\mathrm{C}=\mathrm{C}$ double bond in the $\beta$ - and $\gamma$-positions to the hydroxyl group is not well established. The pyrolysis and oxidation of two such unsaturated alcohols have been studied, i.e. 3-methyl-2-butenol (prenol) and 3-methyl-3-butenol (isoprenol). Experiments at three equivalence ratios, i.e. $\varphi=0.5, \varphi=1.0$ and $\varphi=\infty$ (pyrolysis), were performed using an isothermal jet-stirred quartz reactor at temperatures ranging from 500 to $1100 \mathrm{~K}$, a pressure of $0.107 \mathrm{MPa}$ and a residence time of $2 \mathrm{~s}$. The reactant and product concentrations were quantified using gas chromatography. A kinetic model has been developed using the automatic network generation tool "Genesys". Several important rate coefficients are obtained from new quantum chemical calculations. Overall, there is a good agreement between model calculated mole fraction profiles and experimental data. Reaction path analysis reveals that isoprenol consumption is dominated by a unimolecular reaction to formaldehyde and isobutene. At the applied operating conditions, the equivalence ratio has no effect on the isoprenol conversion profile. Pyrolysis and oxidation of prenol is dominated by radical chemistry, with hydrogen abstractions from prenol forming resonantly stabilized radicals as dominating conversion path. Oxidation and decomposition of the resulting radicals are predicted to form 3-methyl-2-butenal and 2-methyl-1,3-butadiene, which have been detected as important products in the reactor effluent.
\end{abstract}

Keywords: Unsaturated alcohols; Pyrolysis; Oxidation; Kinetic model; Unimolecular decomposition

* corresponding author: kevin.vangeem@ugent.be 


\section{Introduction}

Alcohols are attractive alternative fuels as they can be produced from renewable feedstocks and as their combustion leads to reduced levels of NOx and soot in the exhaust compared to traditional fossil fuels [1]. Ethanol is a first generation bio-derived fuel and the current biofuel market leader. Disadvantages of ethanol, including high hygroscopicity and low energy density, and novel alcohol-production routes from cellulosic biomass have triggered fundamental research towards understanding the combustion chemistry of larger alcohols, i.e. alcohols having four or more carbon atoms. Longer chain alcohols have improved physical properties compared to ethanol. Recent experimental and detailed kinetic modeling studies have highlighted that combustion of such alcohols results in a wide range of products, including ketones, aldehydes and unsaturated alcohols [1-8]. Unsaturated alcohols are important intermediates and understanding their reactivity is of interest to improve kinetic models for alcohol combustion [1]. Besides being intermediates unsaturated alcohols themselves can be considered next-generation bio-derived fuels for spark-ignition combustion engines. Recently, several groups proposed metabolic engineering of Escherichia coli for the highly selective production of prenol and isoprenol from glucose [9-10].

Unsaturated alcohols have both a hydroxyl group and a $\mathrm{C}=\mathrm{C}$ double bond incorporated in their molecular structure. The individual effect of either type of functional group has been investigated previously. The presence and position of a hydroxyl group has an impact on the reactivity of the molecule. Ignition delay times are typically higher for alcohols compared to the analogous alkanes at low temperatures $(<750 \mathrm{~K})$ while they are lower at high temperatures $(<1000 \mathrm{~K})$ [11]. The difference in ignition delay times diminishes as the length of the alkyl chain increases $[5,11]$. The hydroxyl group in alcohols changes the bond dissociation energies (BDE) of nearby $\mathrm{C}-\mathrm{C}$ and $\mathrm{C}-\mathrm{H}$ bonds compared to the analogous alkanes $[12,13]$. Those BDE changes have an impact on, e.g. the rate coefficients of hydrogen abstraction reactions, the main consumption channels of fuels in combustion and pyrolysis. In alcohols, hydrogen abstraction reactions from the $\alpha$-carbon are favored and the resulting radicals react at oxidizing conditions mainly with molecular oxygen forming an aldehyde and a hydroperoxy radical [14-16], which is rather unreactive at low temperature.

The presence and position of a $\mathrm{C}=\mathrm{C}$ double bond in hydrocarbons and its effect on combustion characteristics has been investigated previously [17-19]. The $\mathrm{C}-\mathrm{H}$ bond of the carbon atom in the $\alpha$-position to the $\mathrm{C}=\mathrm{C}$ bond and the $\mathrm{C}-\mathrm{C}$ bond between the carbon atoms in $\alpha$ and $\beta$-positions are weak as scission leads to resonantly stabilized radicals [17]. The resonance stabilization in these radicals is lost upon addition of molecular oxygen, important for autoignition and chain branching at low temperatures, and redissociation of the adduct to the initial reactants is therefore a major reaction channel [20]. The low-temperature reactivity of alkenes is reduced compared to alkanes [19].

Studies regarding the combined effect of both a hydroxyl group and a $\mathrm{C}=\mathrm{C}$ double bond on the reactivity of a molecule have focused on enols [21]. Enols are unsaturated alcohols of the general structure $\mathrm{RC}=\mathrm{C}(\mathrm{R}) \mathrm{OH}$, where a hydroxyl group is attached to a vinylic carbon atom. In the pyrolysis and oxidation of saturated alcohols, they are produced by hydrogen abstraction from the carbon atom in alpha position of the hydroxyl group followed by subsequent $\beta$-scission [1]. They are also formed in the oxidation of hydrocarbons [21,22], for example $\mathrm{C}_{2} \mathrm{H}_{4}+\bullet \mathrm{OH} \rightleftharpoons$ 
- $\mathrm{CH}_{2} \mathrm{CH}_{2} \mathrm{OH} \rightleftharpoons \mathrm{CH}_{2}=\mathrm{CHOH}+\bullet \mathrm{H}[23]$ and $\mathrm{C}_{3} \mathrm{H}_{6}+\bullet \mathrm{OH} \rightleftharpoons \mathrm{CH}_{3} \bullet \mathrm{CHCH}_{2} \mathrm{OH} \rightleftharpoons \mathrm{CH}_{3} \mathrm{CH}=\mathrm{CHOH}+\bullet \mathrm{H}$ [24] and [25]. Enols may isomerize to their corresponding aldehydes by keto-enol tautomerization, which is catalyzed by hydrogen atoms, hydroperoxy radicals [26] and carboxylic acids [27] in the gas phase.

In the past, the reactivity of unsaturated alcohols, in which the hydroxyl group is separated from the $\mathrm{C}=\mathrm{C}$ double bond by one or more carbon atoms, has not received much attention except for studies that focused at atmospheric or low-pressure conditions [28]. Recently, Welz et al. investigated the oxidation of 3-methyl-2-buten-1-ol (prenol) and 3-methyl-3-buten-1-ol (isoprenol), see Figure 1, at $550 \mathrm{~K}$ and low pressure (8 Torr) [29]. In their experiments, chlorine atoms, which were generated from $\mathrm{Cl} 2$ by pulsed laser photolysis, abstract hydrogen from prenol and isoprenol. The radicals reacted with oxygen and the product spectrum was analyzed using multiplexed synchrotron photoionization mass spectrometry. The main products during prenol and isoprenol oxidation were the corresponding aldehydes, 3-methyl-2-butenal and 3-methyl-3butenal respectively. The formation of these aldehydes was supported mechanistically with quantum chemical calculations. The reactions $\mathrm{C}=\mathrm{C}(\mathrm{C}) \mathrm{CC} \bullet \mathrm{OH}+\mathrm{O}_{2} \rightleftharpoons \mathrm{C}=\mathrm{C}(\mathrm{C}) \mathrm{CC}(\mathrm{OO} \bullet) \mathrm{OH} \rightleftharpoons$ $\mathrm{C}=\mathrm{C}(\mathrm{C}) \mathrm{CC}=\mathrm{O}+\mathrm{HO}_{2} \bullet$ and $\mathrm{CC}(\mathrm{C})=\mathrm{CC} \bullet \mathrm{OH}+\mathrm{O}_{2} \rightleftharpoons \mathrm{CC}(\mathrm{C})=\mathrm{CC}(\mathrm{OO} \bullet) \mathrm{OH} \rightleftharpoons \mathrm{CC}(\mathrm{C})=\mathrm{CC}=\mathrm{O}+\mathrm{HO} 2 \bullet$ are the only energetically accessible reaction paths starting from the prenol and isoprenol radicals with $\mathrm{O}_{2}$. Welz et al. concluded that unsaturated alcohols have reduced low-temperature reactivity compared to saturated alcohols, similar to unsaturated and saturated methyl esters [30].

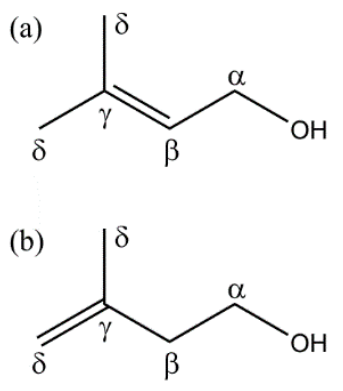

Figure 1: Molecular representation of prenol (a) and isoprenol (b). Greek letters are atom labels which will be used throughout this work.

This work aims at providing a better understanding of the reactivity of unsaturated alcohols in which the double bond is separated from the hydroxyl group by one and two carbon atoms. An extensive experimental dataset was obtained for the oxidation and pyrolysis of prenol and isoprenol in a dedicated jet-stirred reactor. Furthermore, a kinetic model was developed with the automated network generation code Genesys [31]. Several high level theoretical calculations were conducted to describe special reactions that could not be treated automatically. The model calculated mole fraction profiles were compared with the newly obtained experimental data and important reactions were identified. The developed kinetic model should be helpful to improve existing kinetic models for the oxidation and pyrolysis of saturated alcohols.

\section{Experimental setup and procedure}

The applied experimental setup has been extensively described by Herbinet and coworkers [32-34] and only the main features are summarized below. The interested reader is referred to 
the aforementioned literature for additional information. The setup consists of three parts: feed section, reactor section and analysis section.

The feed section ensures a stable, continuous stream of helium, oxygen and prenol or isoprenol to the reactor. Helium and oxygen were provided by Messer (purities of 99.99\% and 99.999\%, respectively). Their flow rates to the reactor were controlled using two gas-mass-flow controllers. Prenol and isoprenol were provided by Sigma Aldrich (purity of 98\%). The flow rate to the reactor was controlled using a liquid-Coriolis-flow-controller. The liquid flow was mixed with helium and passed through an evaporator. The fuel/helium blend was subsequently mixed with oxygen. Prior to entering the reactor, the gaseous mixture is heated to the reactor temperature in an annular pre-heating zone. Heating is provided by Thermocoax resistance wires. The residence time in this zone is short compared to the residence time in the reactor.

The reactor is a spherical jet-stirred reactor made out of quartz. The reactive mixture enters the reactor through four nozzles, positioned in the center of the reactor. The nozzles were designed to ensure thermal homogeneity and avoid concentration gradients inside the reactor. Heating of the reactor is again provided by Thermocoax resistance wires. The reaction temperature is measured by a type $\mathrm{K}$ thermocouple positioned in a glass finger in the center of the reactor. The uncertainty on the temperature reading is approximately $5 \mathrm{~K}$. The pressure in the reactor is controlled using a needle valve positioned downstream of the reactor.

The reactor effluent was analyzed using three dedicated gas chromatographs. The first gas chromatograph is equipped with a thermal conductivity detector and a Carbosphere packed column. It was used to quantify $\mathrm{O} 2, \mathrm{CO}$ and $\mathrm{CO} 2$. The second gas chromatograph is equipped with a flame-ionization detector and a PlotQ capillary column. The flame-ionization detector was preceded by a methanizer to detect $\mathrm{CO}, \mathrm{CO} 2$ and formaldehyde with improved sensitivity. This gas chromatograph was used to quantify molecules containing up to five carbon atoms. The third gas chromatograph is equipped with a flame-ionization detector and a HP-5MS capillary column for quantifying molecules containing at least five carbon atoms. Response factors for $\mathrm{O}_{2}, \mathrm{CO}, \mathrm{CO}_{2}, \mathrm{CH}_{4}$, $\mathrm{C}_{2} \mathrm{H}_{2}, \mathrm{C}_{2} \mathrm{H}_{4}, \mathrm{C}_{2} \mathrm{H}_{6}, \mathrm{C}_{3} \mathrm{H}_{6}, \mathrm{CH}_{2} \mathrm{O}$, prenol, isoprenol, benzene and toluene were determined by injecting calibration mixtures. Response factors for other species were determined using the effective carbon number method.

The exit of the reactor is connected with the aforementioned gas chromatographs through heated transfer lines kept at $473 \mathrm{~K}$ to avoid condensation. Online sampling allowed to obtain reproducible results for the majority of molecules detected in this work. In the case of prenol oxidation and pyrolysis, offline sampling was used to accurately quantify the reactant. During offline sampling, a glass trap, cooled by liquid nitrogen, is connected to the reactor exit. Products exiting the reactor accumulate in the trap. After a known period of time, typically $10 \mathrm{~min}$, the glass trap is disconnected and heated to ambient temperature. Subsequently, a solvent (acetone) and internal standard (n-octane) are added. The resulting mixture is injected into the third gas chromatograph and analyzed. This method proved to be efficient to avoid repeatability problems due to the condensation and/or adsorption of prenol on the walls of the transfer line.

In this work, the prenol and isoprenol inlet mole fraction was kept constant at 0.008 . The inlet volumetric flow rate was fixed at $4.06 \times 10^{-5} \mathrm{~m}^{3} \mathrm{~s}^{-1}$ which corresponds to a residence time of $2 \mathrm{~s}$. The relative uncertainty in gas flow rate is approximately $1 \%$ which results in an uncertainty in 
residence time of $0.02 \mathrm{~s}$. For both unsaturated alcohols, three equivalence ratios were investigated, i.e. $\varphi=0.5,1.0$ and $\infty$. Experiments were performed at temperatures ranging from 500 to $1100 \mathrm{~K}$ and at a constant pressure of $0.107 \mathrm{MPa}$. Several experiments were repeated. The repeatability on experimental mole fractions is approximately $6 \%$ in isoprenol oxidation and pyrolysis and $7 \%$ in prenol oxidation and pyrolysis. The preceding procedure allowed to close the carbon molar balance within $5 \%$. Note that $\mathrm{H}_{2} \mathrm{O}$ was not quantified in this work, therefore, hydrogen and oxygen molar balances could not be verified.

\section{Computational method}

All electronic structure calculations reported in this study were performed with the Gaussian 09 revision D suite of programs [35] as implemented on the high-performance supercomputing facility at Ghent University. The CBS-QB3 level of theory [36] was used exclusively to obtain thermodynamic data for prenol, isoprenol, their radicals and related peroxy species, and to provide input data for transition state theory, which was used to calculate rate expressions. The primary information obtainable from CBS-QB3 calculations are geometries, external moments of inertia, harmonic oscillator frequencies and the electronic energy at $0 \mathrm{~K}$. The first three properties are utilized in statistical mechanics calculations to calculate entropies, heat capacities and thermal contributions to the enthalpy. Except for internal rotations, which were treated separately, all internal modes were assumed to behave as uncoupled harmonic oscillators. A scaling factor of 0.99 was applied as usual. Internal modes that resemble rotations around a single bond were treated separately by replacing the contributions of the corresponding oscillators to the partition function by numerically calculated partition functions of hindered rotors. The required hindrance potentials were obtained from scans, in which the dihedral angle defining the rotation was varied from $0^{\circ}$ to $360^{\circ}$ in steps of $10^{\circ}$ while all other molecule parameters were allowed to optimize. The obtained hindrance potential was then expressed as a Fourier series. Together with the reduced moment of inertia calculated at the I $(2,3)$ level as defined by East and Radom [37], the hindrance potential was used to construct the Schrödinger equation for 1-D rotation. The eigenvalues of the solution to this Schrödinger equation represent the energy levels of this mode. They were used to determine the partial partition function as a function of temperature. After corrections for symmetry and optical isomers, the total partition function was used to calculate the thermal contribution to the enthalpy, standard entropy and temperature-dependent heat capacity data.

Enthalpies of formation were calculated with the atomization method [38]. Two additional corrections accounting for spin-orbit interactions $[39,40]$ and systematic bond corrections (BAC) [39] significantly improve these values as has been shown in previous work [41]. However, such corrections are only needed to calculate the thermodynamic properties. All transition state calculations used uncorrected enthalpy data because BACs are not known for transition states. All data were stored as NASA polynomials.

Transition state theory expressed in terms of Free Gibbs energies was used to calculate the rate coefficients:

$k_{T S T}(T)=\chi(T) \cdot \frac{k_{B} T}{h} \cdot\left(\frac{R T}{p}\right)^{\Delta n-1} \cdot \exp \left(\frac{-\Delta G^{\ddagger}}{R T}\right)$ 
$\Delta G^{\ddagger}$ is the Gibbs free energy difference between transition state without the transitional mode and reactant(s), $\Delta n$ is the molecularity of the reaction ( 2 for bimolecular, 1 for unimolecular reactions), and $\chi(T)$ accounts for quantum mechanical tunneling. All other symbols have their usual meaning. We used the asymmetric Eckart potential to estimate tunneling contributions $\chi(T)$. The Gibbs free energies were obtained from the NASA polynomials. Rate coefficients were calculated for the temperature range $300 \mathrm{~K}$ to $2500 \mathrm{~K}$ in steps of $50 \mathrm{~K}$ and the results were regressed to a modified Arrhenius expression.

The majority of calculated reaction rate coefficients are believed to be within a factor of 3 of experimental data. Rate coefficients of reactions for which hydrogen bonding is important, such as reactions involving peroxy radicals, have a higher uncertainty, a factor of 5 . This is caused by the high uncertainty in the entropy values due to coupling of (internal) rotation and vibration modes which are not fully accounted for by the 1-D hindered rotor calculations.

\section{Kinetic model development}

\subsection{Mechanism construction}

A kinetic model has been developed for the oxidation and pyrolysis of prenol and isoprenol. The prenol and isoprenol submechanism has been generated automatically using Genesys [31]. The latter program produces a CHEMKIN readable input file based on a set of initial molecules, thermochemical databases, and a set of reaction families. Symmetry numbers of molecules and transition states are assigned using the computer code SIGMA [42]. The use of Genesys for the construction of kinetic models has been discussed by Van de Vijver et al. [43]. The main features are summarized below.

Each reaction family in Genesys contains a description of how atoms and bonds in a reactive moiety are rearranged through the course of a reaction. The user can provide constraints to a reaction family to prevent the generation of kinetically insignificant reactions. Each reaction family has to be accompanied with a methodology to assign reaction rate coefficients to reactions belonging to the reaction family. Possible methods include Evans-Polanyi relationship, group additivity for pre-exponential factors and activation energies, and reactivity-structure-based rate rules. The initial set of molecules is iteratively tested whether it is eligible to undergo a reaction family. If a molecule contains the required reactive moiety and passes the molecular constraints, the reaction family is executed and products are generated. The reaction network systematically grows until all initial molecules and generated products are tested against the set of reaction families.

Thermochemical data for molecules and radicals are taken from databases when possible [44-47], otherwise the thermodynamic functions are estimated using Benson's group additivity concept for thermochemistry [48], using recently proposed group additive values and hydrogen bond increments $[41,45,46]$.

The developed kinetic model also includes an isobutene oxidation mechanism by Zhou et al., developed at the Combustion Chemistry Centre at National University of Ireland (Galway) [49]. The isobutene mechanism has been constructed hierarchically. It contains submechanisms for the oxidation of hydrogen, methane, ethane, ethene, propene, methanol, ethanol and acetaldehyde 
[50-52]. Kinetic and thermochemical data were assembled from high-level theoretical calculations and experimental measurements. The isobutene mechanism has been validated over a wide range of experimental data, including flame speed, ignition delays and speciation measurements in a jet-stirred reactor [53].

The first generated kinetic model consisted of (i) the Genesys-generated prenol/isoprenol submechanism and (ii) the isobutene oxidation mechanism by Zhou et al. [49] without modifications. Model calculations highlighted the importance of several reactions for model performance. These reactions include hydrogen abstraction from prenol, $\beta$-scission of primary radicals and unimolecular decomposition of isoprenol to isobutene and formaldehyde. The corresponding reaction rate coefficients have been calculated at the CBS-QB3 level of theory and were implemented in the final kinetic model. These reactions are listed in Table 1. Furthermore, the preliminary version of the kinetic model severely underpredicted observed acetaldehyde profiles. Reaction path analysis revealed that this was linked to hydrogen abstraction from ethenol by hydroxyl radicals forming vinoxy and water. The final kinetic model incorporates the complete isobutene oxidation mechanism developed by Zhou et al. [49], including $\mathrm{C}_{0}-\mathrm{C}_{3}$ base, however reaction rate coefficients for hydrogen abstraction from ethenol by hydroxyl radicals forming vinoxy and water were replaced with those proposed by Paraskevas et al. [54] which resulted in satisfactory model performance, as will be shown later.

Table 1: Rate coefficients for selected reactions in the prenol and isoprenol kinetic model. Units: $A T^{n}\left[\mathrm{~s}^{-1}\right.$ monomolecular or $\mathrm{m}^{3} \mathrm{~mol}^{-1} \mathrm{~s}^{-1}$ bimolecular], $E a\left[\mathrm{~kJ} \mathrm{~mol}{ }^{-1}\right], k=A T^{n} \exp (-E a / R T)$ [ $\mathrm{s}^{-1}$ monomolecular or $\mathrm{m}^{3} \mathrm{~mol}^{-1} \mathrm{~s}^{-1}$ bimolecular].

\begin{tabular}{|c|c|c|c|c|c|}
\hline No. & Reaction & $A$ & $n$ & Ea & Reference/Note \\
\hline & Unimolecular decomposition reactions & & & & \\
\hline 1 & Isoprenol $\rightleftarrows \mathrm{C} 2 \mathrm{CC}+\mathrm{CH} 2 \mathrm{O}$ & $7.31 \mathrm{E}+05$ & 1.68 & 145.4 & CBS-QB3 \\
\hline 2 & $\mathrm{C} 2 \cdot \mathrm{CC}+\bullet \mathrm{CH} 2 \mathrm{OH} \rightleftarrows$ Isoprenol & $1.00 \mathrm{E}+07$ & 0.00 & 0.00 & Estimated \\
\hline \multirow[t]{3}{*}{3} & $\mathrm{C} 2 \mathrm{CCC} \bullet+\bullet \mathrm{OH} \rightleftarrows$ Prenol & $2.50 \mathrm{E}+07$ & 0.00 & 0.00 & Estimated \\
\hline & Hydrogen abstraction from unsaturated alcohols & & & & \\
\hline & By hydroxyl radicals & & & & \\
\hline 4 & $\bullet \mathrm{OH}+$ Isoprenol $\rightleftarrows \mathrm{H} 2 \mathrm{O}+\mathrm{CC}(\mathrm{C}) \mathrm{CC} \bullet \mathrm{OH}$ & $5.10 \mathrm{E}-02$ & 2.60 & -133.0 & {$[54]^{\mathrm{a}}$} \\
\hline 5 & $\cdot \mathrm{OH}+$ Isoprenol $\rightleftarrows \mathrm{H} 2 \mathrm{O}+\mathrm{CC}(\mathrm{C}) \mathrm{C} \cdot \mathrm{COH}$ & $1.50 \mathrm{E}-01$ & 2.40 & -3.9 & {$[54]^{\mathrm{b}}$} \\
\hline 6 & $\bullet \mathrm{OH}+$ Isoprenol $\rightleftarrows \mathrm{H} 2 \mathrm{O}+\mathrm{CC}(\mathrm{C} \bullet) \mathrm{CCOH}$ & $7.80 \mathrm{E}-01$ & 2.30 & -5.7 & {$[54]^{\mathrm{c}}$} \\
\hline 7 & $\bullet \mathrm{OH}+$ Prenol $\rightleftarrows \mathrm{H} 2 \mathrm{O}+\mathrm{CC}(\mathrm{C}) \mathrm{CC} \bullet \mathrm{OH}$ & $2.30 \mathrm{E}-02$ & 2.60 & -8.7 & {$[54]^{\mathrm{d}}$} \\
\hline \multirow[t]{2}{*}{8} & $\cdot \mathrm{OH}+$ Prenol $\rightleftarrows \mathrm{H} 2 \mathrm{O}+\mathrm{CC}(\mathrm{C}) \mathrm{C} \cdot \mathrm{COH}$ & $1.56 \mathrm{E}+00$ & 2.30 & -5.7 & {$[54]^{\mathrm{a}}$} \\
\hline & By $\mathrm{H}$ atoms & & & & \\
\hline 9 & $\mathrm{H} \bullet+$ Isoprenol $\rightleftarrows \mathrm{H} 2+\mathrm{CC}(\mathrm{C}) \mathrm{CC} \bullet \mathrm{OH}$ & $8.52 \mathrm{E}-01$ & 2.42 & 17.5 & CBS-QB3 \\
\hline 10 & $\mathrm{H} \bullet+$ Isoprenol $\rightleftarrows \mathrm{H} 2+\mathrm{CC}(\mathrm{C}) \mathrm{C} \bullet \mathrm{COH}$ & $7.90 \mathrm{E}-01$ & 2.45 & 14.6 & CBS-QB3 \\
\hline 11 & $\mathrm{H} \bullet+$ Isoprenol $\rightleftarrows \mathrm{H} 2+\mathrm{CC}(\mathrm{C} \bullet) \mathrm{CCOH}$ & $2.23 \mathrm{E}+00$ & 2.30 & 17.7 & CBS-QB3 \\
\hline 12 & $\mathrm{H} \bullet+$ Prenol $\rightleftarrows \mathrm{H} 2+\mathrm{CC}(\mathrm{C}) \mathrm{CC} \bullet \mathrm{OH}$ & $1.19 E+03$ & 1.58 & 9.2 & CBS-QB3 \\
\hline \multirow[t]{2}{*}{13} & $\mathrm{H} \bullet+$ Prenol $\rightleftarrows \mathrm{H} 2+\mathrm{CC}(\mathrm{C}) \mathrm{C} \bullet \mathrm{COH}$ & $3.50 \mathrm{E}+00$ & 2.31 & 18.0 & CBS-QB3 \\
\hline & By methyl radicals & & & & \\
\hline 14 & $\cdot \mathrm{CH} 3+$ Isoprenol $\rightleftarrows \mathrm{CH} 4+\mathrm{CC}(\mathrm{C}) \mathrm{CC} \bullet \mathrm{OH}$ & $1.63 \mathrm{E}-05$ & 3.44 & 31.1 & CBS-QB3 \\
\hline 15 & $\cdot \mathrm{CH} 3+$ Isoprenol $\rightleftarrows \mathrm{CH} 4+\mathrm{CC}(\mathrm{C}) \mathrm{C} \cdot \mathrm{COH}$ & $4.80 \mathrm{E}-06$ & 3.62 & 23.4 & CBS-QB3 \\
\hline 16 & $\cdot \mathrm{CH} 3+$ Isoprenol $\rightleftarrows \mathrm{CH} 4+\mathrm{CC}(\mathrm{C} \bullet) \mathrm{CCOH}$ & $3.60 \mathrm{E}-05$ & 3.33 & 29.0 & CBS-QB3 \\
\hline 17 & $\bullet \mathrm{CH} 3+$ Prenol $\rightleftarrows \mathrm{CH} 4+\mathrm{CC}(\mathrm{C}) \mathrm{C} \bullet \mathrm{COH}$ & $3.50 \mathrm{E}-05$ & 3.37 & 27.2 & CBS-QB3 \\
\hline \multirow[t]{2}{*}{18} & $\cdot \mathrm{CH} 3+$ Prenol $\rightleftarrows \mathrm{CH} 4+\mathrm{CC}(\mathrm{C}) \mathrm{CC} \bullet \mathrm{OH}$ & $5.56 \mathrm{E}-04$ & 3.01 & 20.7 & CBS-QB3 \\
\hline & By hydroperoxy radicals & & & & \\
\hline 19 & $\mathrm{HO} 2 \bullet+$ Isoprenol $\rightleftarrows \mathrm{H} 2 \mathrm{O} 2+\mathrm{CC}(\mathrm{C}) \mathrm{CC} \bullet \mathrm{OH}$ & $2.46 \mathrm{E}-11$ & 5.26 & 31.3 & {$[64]^{\mathrm{a}}$} \\
\hline
\end{tabular}


Table 1 (continued).

\begin{tabular}{|c|c|c|c|c|}
\hline $20 \mathrm{HO} 2 \bullet+$ Isoprenol $\rightleftarrows \mathrm{H} 2 \mathrm{O} 2+\mathrm{CC}(\mathrm{C}) \mathrm{C} \bullet \mathrm{COH}$ & $5.12 \mathrm{E}-08$ & 4.40 & 56.7 & {$[55]^{c}$} \\
\hline $21 \mathrm{HO} 2 \bullet+$ Isoprenol $\rightleftarrows \mathrm{H} 2 \mathrm{O} 2+\mathrm{CC}(\mathrm{C} \bullet) \mathrm{CCOH}$ & $7.68 \mathrm{E}-08$ & 4.40 & 56.7 & {$[55]^{\mathrm{c}}$} \\
\hline $22 \mathrm{HO} 2 \bullet+$ Prenol $\rightleftarrows \mathrm{H} 2 \mathrm{O} 2+\mathrm{CC}(\mathrm{C}) \mathrm{CC} \bullet \mathrm{OH}$ & $2.70 \mathrm{E}-07$ & 3.79 & 24.3 & CBS-QB3 \\
\hline \multicolumn{5}{|l|}{ By molecular oxygen } \\
\hline $24 \mathrm{O} 2+$ Isoprenol $\rightleftarrows \mathrm{HO} 2 \bullet+\mathrm{CC}(\mathrm{C}) \mathrm{CC} \bullet \mathrm{OH}$ & $2.00 \mathrm{E}+07$ & 0.00 & 198.6 & {$[80]^{\mathrm{e}}$} \\
\hline $25 \mathrm{O} 2+$ Isoprenol $\rightleftarrows \mathrm{HO} 2 \bullet+\mathrm{CC}(\mathrm{C}) \mathrm{C} \bullet \mathrm{COH}$ & $2.00 \mathrm{E}+07$ & 0.00 & 144.5 & {$[80]^{\mathrm{e}}$} \\
\hline $26 \mathrm{O} 2+$ Isoprenol $\rightleftarrows \mathrm{HO} 2 \bullet+\mathrm{CC}(\mathrm{C} \bullet) \mathrm{CCOH}$ & $3.00 \mathrm{E}+07$ & 0.00 & 164.4 & {$[80]^{\mathrm{e}}$} \\
\hline $27 \mathrm{O} 2+$ Prenol $\rightleftarrows \mathrm{HO} 2 \bullet+\mathrm{CC}(\mathrm{C}) \mathrm{CC} \bullet \mathrm{OH}$ & $2.00 \mathrm{E}+07$ & 0.00 & 131.3 & {$[80]^{\mathrm{e}}$} \\
\hline \multicolumn{4}{|l|}{ Radical decomposition } & {$[80]^{\mathrm{e}}$} \\
\hline $29 \mathrm{CC}(\mathrm{C}) \mathrm{CC} \cdot \mathrm{OH} \rightleftarrows \mathrm{CC} \cdot \mathrm{C}+\mathrm{CCOH}$ & $3.07 \mathrm{E}+14$ & 0.00 & 147.7 & {$[59]^{\mathrm{f}}$} \\
\hline $30 \mathrm{CC}(\mathrm{C}) \mathrm{CC} \bullet \mathrm{OH} \rightleftarrows \mathrm{CC}(\mathrm{C} \bullet) \mathrm{CCOH}$ & $9.00 \mathrm{E}+11$ & 0.00 & 109.7 & {$[65,81]^{\mathrm{f}}$} \\
\hline $31 \mathrm{CC}(\mathrm{C}) \mathrm{CC}+\bullet \mathrm{OH} \rightleftarrows \mathrm{CC}(\mathrm{C}) \mathrm{C} \cdot \mathrm{COH}$ & $1.30 \mathrm{E}-02$ & 2.55 & -24.3 & CBS-QB3 \\
\hline $32 \mathrm{CC}(\mathrm{C} \bullet) \mathrm{CCOH} \rightleftarrows \mathrm{CCC}+\mathrm{C} \cdot \mathrm{COH}$ & $3.11 \mathrm{E}+15$ & 0.00 & 236.8 & {$[59]^{\mathrm{f}}$} \\
\hline $33 \mathrm{CC}(\mathrm{C}) \mathrm{CC} \bullet \mathrm{OH} \rightleftarrows \mathrm{CC}(\mathrm{C}) \mathrm{CCO}+\mathrm{H} \bullet$ & $6.40 \mathrm{E}+10$ & 1.03 & 179.0 & CBS-QB3 \\
\hline $34 \mathrm{CC}(\mathrm{C}) \mathrm{CC} \cdot \mathrm{OH} \rightleftarrows \mathrm{CC}(\mathrm{C}) \mathrm{C} \cdot \mathrm{COH}$ & $2.30 \mathrm{E}+06$ & 1.95 & 149.0 & CBS-QB3 \\
\hline \multicolumn{5}{|c|}{ Hydroxyl addition and subsequent reaction channels } \\
\hline 34 Prenol+• $\mathrm{OH} \rightleftarrows \mathrm{C} 2 \mathrm{C}(\mathrm{OH}) \mathrm{C} \cdot \mathrm{COH}$ & $7.80 \mathrm{E}+01$ & 1.29 & -11.1 & {$[24]^{c}$} \\
\hline $35 \mathrm{C} 2 \mathrm{C}(\mathrm{OH}) \mathrm{C} \cdot \mathrm{COH} \rightleftarrows \mathrm{C} 2 \mathrm{C}(\mathrm{OH}) \mathrm{CC}+\bullet \mathrm{OH}$ & $3.00 \mathrm{E}+13$ & 0.00 & 112.1 & a \\
\hline $36 \mathrm{C} 2 \mathrm{C}(\mathrm{OH}) \mathrm{C} \cdot \mathrm{COH} \rightleftarrows \cdot \mathrm{CH} 3+\mathrm{CC}(\mathrm{OH}) \mathrm{CCOH}$ & $9.39 E+13$ & 0.00 & 124.4 & {$[59]^{\mathrm{f}}$} \\
\hline $37 \mathrm{O} 2+\mathrm{C} 2 \mathrm{C}(\mathrm{OH}) \mathrm{C} \cdot \mathrm{COH} \rightleftarrows \mathrm{C} 2 \mathrm{C}(\mathrm{OH}) \mathrm{C}(\mathrm{OO} \bullet) \mathrm{COH}$ & $1.51 \mathrm{E}+09$ & -0.92 & -0.5 & {$[66]^{\mathrm{g}}$} \\
\hline $38 \mathrm{C} 2 \mathrm{C}(\mathrm{OH}) \mathrm{C}(\mathrm{OO} \bullet) \mathrm{COH} \rightleftarrows \mathrm{C} 2 \mathrm{C}(\mathrm{O} \bullet) \mathrm{C}(\mathrm{OOH}) \mathrm{COH}$ & $2.91 \mathrm{E}+12$ & -0.23 & 93.3 & {$[71]^{\mathrm{h}}$} \\
\hline $39 \mathrm{C} 2 \mathrm{C}(\mathrm{O} \bullet) \mathrm{C}(\mathrm{OOH}) \mathrm{COH} \rightleftarrows \mathrm{CC}(\mathrm{O}) \mathrm{C}+\mathrm{C}(\mathrm{O}) \mathrm{COH}+\bullet \mathrm{OH}$ & $6.00 \mathrm{E}+13$ & 0.22 & 59.0 & $\mathrm{i}$ \\
\hline $40 \mathrm{C} 2 \mathrm{C}(\mathrm{OH}) \mathrm{C}(\mathrm{OO} \bullet) \mathrm{COH} \rightleftarrows \mathrm{C} 2 \mathrm{C}(\mathrm{OH}) \mathrm{C}(\mathrm{OOH}) \mathrm{CO} \bullet$ & $2.91 \mathrm{E}+12$ & -0.23 & 93.3 & {$[71]^{\mathrm{h}}$} \\
\hline 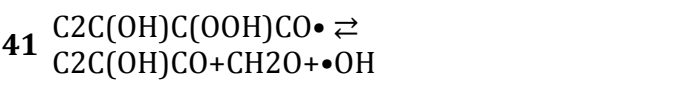 & $6.00 \mathrm{E}+13$ & 0.22 & 59.0 & $\mathrm{i}$ \\
\hline 42 Prenol+・ $\mathrm{OH} \rightleftarrows \mathrm{C} 2 \mathrm{C} \bullet \mathrm{C}(\mathrm{OH}) \mathrm{COH}$ & $7.80 \mathrm{E}+01$ & 1.29 & -11.1 & {$[24]^{\mathrm{c}}$} \\
\hline $43 \mathrm{C} 2 \mathrm{C} \cdot \mathrm{C}(\mathrm{OH}) \mathrm{COH} \rightleftarrows \mathrm{C} 2 \mathrm{CCOH}+\bullet \mathrm{CH} 2 \mathrm{OH}$ & $7.68 \mathrm{E}+13$ & 0.00 & 127.6 & CBS-QB3 \\
\hline $44 \mathrm{O} 2+\mathrm{C} 2 \mathrm{C} \bullet \mathrm{C}(\mathrm{OH}) \mathrm{COH} \rightleftarrows \mathrm{C} 2 \mathrm{C}(\mathrm{OO} \bullet) \mathrm{C}(\mathrm{OH}) \mathrm{COH}$ & $4.95 \mathrm{E}+05$ & 0.40 & -3.4 & {$[66]^{\mathrm{g}}$} \\
\hline $45 \mathrm{C} 2 \mathrm{C}(\mathrm{OO} \bullet) \mathrm{C}(\mathrm{OH}) \mathrm{COH} \rightleftarrows \mathrm{C} 2 \mathrm{C}(\mathrm{OOH}) \mathrm{C}(\mathrm{O} \bullet) \mathrm{COH}$ & $2.91 \mathrm{E}+12$ & -0.23 & 93.3 & {$[71]^{\mathrm{h}}$} \\
\hline \multicolumn{5}{|c|}{ Reactions of prenol radicals with molecular oxygen } \\
\hline $47 \mathrm{O} 2+\mathrm{CC}(\mathrm{C}) \mathrm{CC} \bullet \mathrm{OH} \rightleftarrows \mathrm{CC}(\mathrm{C}) \mathrm{CC}(\mathrm{OO} \bullet) \mathrm{OH}$ & $2.90 \mathrm{E}+01$ & 1.59 & 4.5 & {$[67]^{\mathrm{c}}$} \\
\hline $48 \mathrm{CC}(\mathrm{C}) \mathrm{CC}(\mathrm{OO} \bullet) \mathrm{OH} \rightleftarrows \mathrm{CC}(\mathrm{C}) \mathrm{CCO}+\mathrm{HO} 2 \bullet$ & $1.20 \mathrm{E}+12$ & 0.20 & 41.0 & CBS-QB3 \\
\hline $49 \mathrm{O} 2+\mathrm{CC}(\mathrm{C}) \mathrm{C} \cdot \mathrm{COH} \rightleftarrows \bullet \mathrm{OOCC}(\mathrm{C}) \mathrm{CCOH}$ & $2.90 \mathrm{E}+01$ & 1.59 & 4.5 & {$[67]^{c}$} \\
\hline $5 \mathbf{5 0} \bullet \mathrm{OOCC}(\mathrm{C}) \mathrm{CCOH} \rightleftarrows \mathrm{HOOCC}(\mathrm{C}) \mathrm{CC} \bullet \mathrm{OH}$ & $4.48 \mathrm{E}+06$ & 1.29 & 59.6 & {$[68]^{\mathrm{j}}$} \\
\hline $51 \mathrm{O} 2+\mathrm{HOOCC}(\mathrm{C}) \mathrm{CC} \bullet \mathrm{OH} \rightleftarrows \mathrm{HOOCC}(\mathrm{C}) \mathrm{CC}(\mathrm{OO} \bullet) \mathrm{OH}$ & $2.90 \mathrm{E}+01$ & 1.59 & 4.5 & {$[67]^{\mathrm{c}}$} \\
\hline 52 HOOCC $(\mathrm{C}) \mathrm{CC}(\mathrm{OO} \bullet) \mathrm{OH} \rightleftarrows \mathrm{HOOCC}(\mathrm{C}) \mathrm{CCO}+\mathrm{HO} 2 \bullet$ & $1.20 \mathrm{E}+12$ & 0.20 & 41.0 & $\mathrm{k}$ \\
\hline
\end{tabular}

a Generated using Genesys, reaction rate coefficient is estimated from the analogous ethanol/2-hydroxy-ethyl reaction.

$\mathrm{b}$ Generated using Genesys, reaction rate coefficient is estimated from the analogous 1-butene reaction.

c Generated using Genesys, reaction rate coefficient is estimated from the analogous propene/allyl reaction.

$\mathrm{d}$ Generated using Genesys, reaction rate coefficient is estimated from the analogous 2-propen-1-ol reaction.

e Generated using Genesys, reaction rate coefficient is estimated using Evans-Polanyi relationship.

$\mathrm{f}$ Generated using Genesys, reaction rate coefficient is estimated using group additivity method.

$\mathrm{g}$ Generated using Genesys, reaction rate coefficient is estimated from the analogous alkyl reaction.

$\mathrm{h}$ Generated using Genesys, reaction rate coefficient is estimated from the analogous isobutene/isobutenyl reaction.

${ }^{\mathrm{i}}$ Generated using Genesys, reaction rate coefficient is estimated from the analogous acetone/2-oxy-propane reaction.

j Generated using Genesys, reaction rate coefficient is estimated from the analogous 2-pentene reaction.

$\mathrm{k}$ Generated using Genesys, reaction rate coefficient is estimated from the analogous prenol reaction. 
The following paragraphs focuses more detailed on three essential aspects of the kinetic model, i.e. the utilized reaction families in Genesys, the unimolecular decomposition of isoprenol to isobutene and formaldehyde and thermochemistry of prenol peroxy radicals.

\subsection{Reaction families}

Genesys requires the user to define all reaction families that shall be considered in the network generation process. The reaction families utilized in this work are listed in Table 2. Reaction rate coefficients for these families were taken from various sources including estimation techniques, such as group additivity, Evans-Polanyi, reactivity-structure-based rate rules, or analogy of known reactions.

Table 2: Reaction families considered in this work.

Reaction family

Hydrogen abstraction

Intermolecular

by C•

by $\mathrm{H} \bullet$

by $\bullet \mathrm{OH}$

by $\mathrm{HO} 2 \bullet$

by 02

Intramolecular

$\mathrm{C}-(\mathrm{C}) \mathrm{n}-\mathrm{C} \cdot \rightleftarrows \cdot \mathrm{C}-(\mathrm{C}) \mathrm{n}-\mathrm{C}$

$\mathrm{C}-(\mathrm{C}) \mathrm{n}-\mathrm{O}-\mathrm{O} \bullet \rightleftarrows \bullet \mathrm{C}-(\mathrm{C}) \mathrm{n}-\mathrm{O}-\mathrm{OH}$

$\mathrm{C}(\mathrm{O}-\mathrm{O} \bullet)-\mathrm{C}-\mathrm{OH} \rightleftarrows \mathrm{C}(\mathrm{O}-\mathrm{OH})-\mathrm{C}-\mathrm{O} \bullet$

$\beta$-scission/radical addition

Carbon centered radicals

-C-C-C $\rightleftarrows \mathrm{CC}+\bullet \mathrm{C}$

$\bullet \mathrm{C}-\mathrm{C}-\mathrm{H} \rightleftarrows \mathrm{CC}+\bullet \mathrm{H}$

-C-C-OH $\rightleftarrows \mathrm{CC}+\bullet \mathrm{OH}$

-C-O-H $\rightleftarrows \mathrm{CO}+\bullet \mathrm{H}$

-C-C-O-OH $\rightleftarrows \mathrm{CC}+\mathrm{HO}^{\bullet}$

Oxygen centered radicals

$\bullet \mathrm{O}-\mathrm{C}-\mathrm{C} \rightleftarrows \mathrm{CO}+\bullet \mathrm{C}$

$\bullet \mathrm{O}-\mathrm{C}-\mathrm{H} \rightleftarrows \mathrm{CO}+\bullet \mathrm{H}$

$\alpha$-scission

CO $\alpha$-scission

$\mathrm{C}-\mathrm{C}(\mathrm{O}) \bullet \rightleftarrows \mathrm{C} \bullet+\mathrm{CO}$

Concerted reaction paths

Peroxy-alkyl to alkene and HO2

$\mathrm{C}-\mathrm{C}-\mathrm{O}-\mathrm{O} \bullet \rightleftarrows \mathrm{CC}+\mathrm{HO} 2 \bullet$

Hydroperoxy-alkene to cyclic ether and $\mathrm{OH}$

$\cdot \mathrm{C}-(\mathrm{C}) \mathrm{n}-\mathrm{O}-\mathrm{OH} \rightleftarrows \mathrm{cy}(\mathrm{C}-(\mathrm{C}) \mathrm{n}-\mathrm{O})+\bullet \mathrm{OH}$

Oxidation of $\alpha$-hydroxyalkyl

$\mathrm{C}(-\mathrm{O}-\mathrm{O} \bullet)-\mathrm{OH}+\mathrm{O} 2 \rightleftarrows \mathrm{CO}+\mathrm{HO} 2 \bullet$

Radical++02

Oxygen addition to carbon-centered radicals

$\mathrm{C} \bullet+02 \rightleftarrows \mathrm{C}-\mathrm{O}-\mathrm{O} \bullet$

Scission/radical recombination

Recombination allylic radicals with $\mathrm{HO} 2$

-C $-\mathrm{CC}+\mathrm{HO} 2 \bullet \rightleftarrows \mathrm{C}-\mathrm{CC}-\mathrm{O}-\mathrm{OH}$

Scission of $\mathrm{O}-\mathrm{O}$ bond

$\mathrm{C}-\mathrm{O}-\mathrm{OH} \rightleftarrows \mathrm{C}-\mathrm{O} \bullet+\bullet \mathrm{OH}$ 
For several reaction families, only a limited number of kinetics studies are available in literature. Reaction rate coefficients of a reaction belonging to such a reaction family are assigned based on analogy to similar hydrocarbon/alcohol reactions. For example, reaction rate coefficients for hydrogen abstraction by hydroperoxy radicals from the $\delta$-carbon atom in prenol were taken from a theoretical study by Zador et al. regarding the reactions of propene with hydroperoxy [55] (cf. reaction no. (23) in Table 1 and Figure 2). The pre-exponential factor of hydrogen abstraction by a hydroperoxy radical from the $\delta$-carbon atom in prenol is a factor two higher than hydrogen abstraction by a hydroperoxy radical from the allylic carbon atom in propene, given the increase in number of single-events.

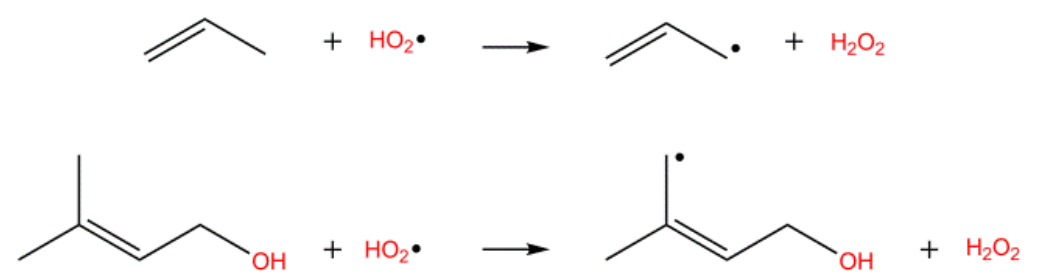

Figure 2: Hydrogen abstraction by hydroperoxy forming resonantly stabilized radicals.

The sources of rate expressions for a selected number of reaction families are discussed in the following paragraphs. All reaction families, along with details regarding reaction rate coefficients, are listed in Supplementary Material.

\subsubsection{Hydrogen abstraction and radical decomposition reactions}

Reaction rate coefficients of reactions belonging to the reaction families (i) hydrogen abstraction by carbon-centered radicals, (ii) hydrogen abstraction by hydrogen atoms, (iii) carbon-centered $\beta$-scission/carbon-centered radical addition and (iv) hydrogen-centered $\beta$-scission/hydrogen atom addition were calculated using the group additive databases developed by Marin and coworkers [56-59]. These authors applied Benson's group additivity concept to transition state theory and derived group additive values from CBS-QB3 calculations at the high-pressure limit. If group values were not available, e.g. for hydrogen abstraction from the carbonyl group in aldehydes, reaction rate coefficients were taken from literature, e.g. Mendes et al. [60].

Reaction rate coefficients for hydrogen abstraction by hydroxyl radicals from primary/secondary/tertiary $\mathrm{C}-\mathrm{H}$ moieties forming alkyl radicals are provided by an experimental study by Sivaramakrishnan et al. [61] and Badra et al. [62]. If hydrogen abstraction by a hydroxyl radical forms a resonantly stabilized radical, reaction rate coefficients are taken from the analogous alkene reactions [54]. Reaction rate coefficients for hydrogen abstraction by a hydroxyl radical from a carbon atom connected to a $\mathrm{C}=\mathrm{C}$ double bond and a hydroxyl group are assumed to be analogous to hydrogen abstraction by hydroxyl from allyl alcohol [54].

Reaction rate coefficients for hydrogen abstraction by hydroperoxy radicals from primary/secondary/tertiary carbon atoms forming alkyl radicals are provided by a theoretical study by Aguilera-Iparraguirre et al. [63]. If hydrogen abstraction by hydroperoxy forms an $\alpha$ hydroxy-alkyl radical or a resonantly stabilized radical, reaction rate coefficients are taken from the analogous ethanol $\left(\mathrm{HO}_{2} \bullet+\mathrm{CH}_{3} \mathrm{CH}_{2} \mathrm{OH} \rightleftarrows \mathrm{H}_{2} \mathrm{O}_{2}+\mathrm{CH}_{3} \mathrm{CH}(\bullet) \mathrm{OH}\right)$ [64] or propene $\left(\mathrm{HO}_{2} \bullet+\right.$ $\mathrm{CH}_{3} \mathrm{CH}=\mathrm{CH}_{2} \rightleftarrows \mathrm{H}_{2} \mathrm{O}_{2}+\cdot \mathrm{CH}_{2} \mathrm{CH}=\mathrm{CH}_{2}$ ) [55] reactions. 
Intramolecular hydrogen abstractions proceed through a cyclic transition state. The activation energy of such reactions can be considered to consist of two components, the ring strain induced by the transition state and the activation energy of the analogous bimolecular hydrogen abstraction reaction [65]. The pre-exponential factor is correlated with the loss of hindered rotors in the transition state [65]. In this work, rate coefficients for intramolecular hydrogen abstraction reactions of carbon-centered radicals are estimated using the reactivity-structure-based rate rules proposed by Wang et al. [65].

\subsubsection{Low temperature oxidation pathways}

Bugler et al. recently recommended rate rules for oxygen addition to alkyl radicals ( $\mathrm{R}+\mathrm{O}_{2} \rightleftarrows \mathrm{ROO}$ ), concerted decomposition of peroxy radicals to alkenes and hydroperoxy ( $\mathrm{ROO} \rightleftarrows$ alkene $+\mathrm{HO}_{2}$ ), intramolecular hydrogen abstraction of peroxy radicals (ROO $\rightleftarrows \mathrm{QOOH}), \beta$-scission of $\beta$-hydroperoxy-alkyl radicals to alkenes and hydroperoxy $\left(\beta-\mathrm{QOOH} \rightleftarrows\right.$ alkene $+\mathrm{HO}_{2}$ ) and decomposition of hydroperoxy-alkyl radicals to cyclic ethers and hydroxyl $(\mathrm{QOOH} \rightleftarrows$ cyclic ether $+\mathrm{OH})$ [66]. These reaction pathways were implemented in this work. For all reaction families mentioned above, reaction rate coefficients were taken from Bugler et al. in the case of alkyl radicals [66] or from the analogous alkene system in the case of resonantly stabilized radicals $[67,68]$. For example, reaction rate coefficients for oxygen addition on resonantly stabilized radicals were taken from oxygen addition on allyl [67].

\subsubsection{Reaction pathways characteristic for alkene and alcohol oxidation}

Alkenes and alcohols have several characteristic oxidation pathways. Resonantly stabilized radicals, such as allyl radicals, have a relatively long life time compared to alkyl radicals. Recombination with hydroperoxy radicals therefore is an important consumption channel, as shown for allyl in propene oxidation [50] and 2-methyl-butenyl in 2-methyl-2-butene oxidation [69]. The resulting hydroperoxide has a weak $0-0$ bond and scission results in a hydroxyl radical and an oxy radical. Rate coefficients for recombination reactions of resonantly stabilized radicals with hydroperoxy and associated reactions were taken from the theoretical study by Goldsmith et al. on the reactions of allyl with hydroperoxy [70].

One of the most important reaction pathways of hydroxyl radicals, the main chain carrying radical at low-temperatures, is addition to the $\mathrm{C}=\mathrm{C}$ double bond of alkenes, forming a $\beta$-hydroxy-alkyl radical. Oxygen addition to this radical yields a hydroxyl-alkyl-peroxy radical. Intramolecular hydrogen abstraction of the latter radical, in which the hydrogen of the hydroxyl group migrates to the peroxy group, has a relatively low energy barrier [71]. The formed hydroperoxy-alkoxy radical dissociates by $\mathrm{C}-\mathrm{O} \beta$-scission forming a $\alpha$-hydroperoxy-keto-alkyl radical. It is unstable and decomposes rapidly by $0-0 \beta$-scission. The described reaction sequence, known as the Waddington reaction, plays an important role in the oxidation of alkenes [18]. Reaction rate coefficients were estimated from a theoretical study by Sun et al. [71].

Oxidation of alcohols has a high selectivity to aldehydes. The reaction of molecular oxygen with an $\alpha$-hydroxyalkyl radical to hydroperoxy and the corresponding aldehyde has a low energy barrier $[14,72]$. Therefore, this reaction family was included in the current work. Reaction rate coefficients were taken from a theoretical study by da Silva et al. on the reaction of $\alpha$-hydroxyethyl 
with molecular oxygen [14]. The latter reaction rate coefficients have also been adopted in recent kinetic models for alcohol oxidation proposed by Sarathy and co-workers $[1,5-7,13,73]$.

\subsection{Isoprenol molecular decomposition}

As will be discussed in the results, isoprenol oxidation and pyrolysis have a high selectivity to isobutene and formaldehyde. The molecular decomposition of isoprenol to isobutene and formaldehyde has been investigated at the CBS-QB3 level of theory, using the methodology described above. This reaction is analogous to the retro-ene reaction of alkenes [18] and proceeds through a six-membered transition state, in which the hydrogen atom of the hydroxyl group bridges to the $\mathrm{C}=\mathrm{C}$ double bond, as illustrated in Figure 3. The retro-ene reaction family is known to be an important unimolecular consumption channel of olefins and unsaturated methyl esters $[34,74]$. The rate coefficient for the reaction of isoprenol to isobutene and formaldehyde is displayed in Figure 3 as a function of temperature, and the retro-ene reaction of 1-pentene to propene and ethene has been added for comparison. At $500 \mathrm{~K}$ and $1000 \mathrm{~K}$, the rate coefficients for the reaction isoprenol to isobutene and formaldehyde are, respectively, $1 \mathrm{E}+7$ and $3 \mathrm{E}+2$ times faster than the retro-ene reaction of 1-pentene to propene and ethene (reaction rate coefficients calculated at the CBS-QB3 level of theory). The large reactivity differences are caused by substantially different activation energies as shown in Figure 3. The activation energy for the reaction isoprenol to isobutene and formaldehyde is $159 \mathrm{~kJ} \mathrm{~mol}^{-1}$, the activation energy for the reaction 1-pentene to propene and ethene is $236 \mathrm{~kJ} \mathrm{~mol}^{-1}$. In the case of isoprenol to isobutene and formaldehyde, a hydrogen atom moves from an $\mathrm{O}-\mathrm{H}$ moiety to a $\mathrm{C}=\mathrm{C}$ double bond. Given the difference in electronegativities between oxygen and hydrogen atoms, the migrating hydrogen atom is positively charged. As the $\mathrm{C}=\mathrm{C}$ double bond contains a high electron density, polarization lead to stabilization of the transition state. Such an effect is not present in the retro-ene reaction of 1-pentene. Furthermore, the HOMO-LUMO energy gap is important in pericyclic reactions as the electrons rearrange by using empty orbitals. Those energetics depend on different factors, including the presence of hetero-atoms.

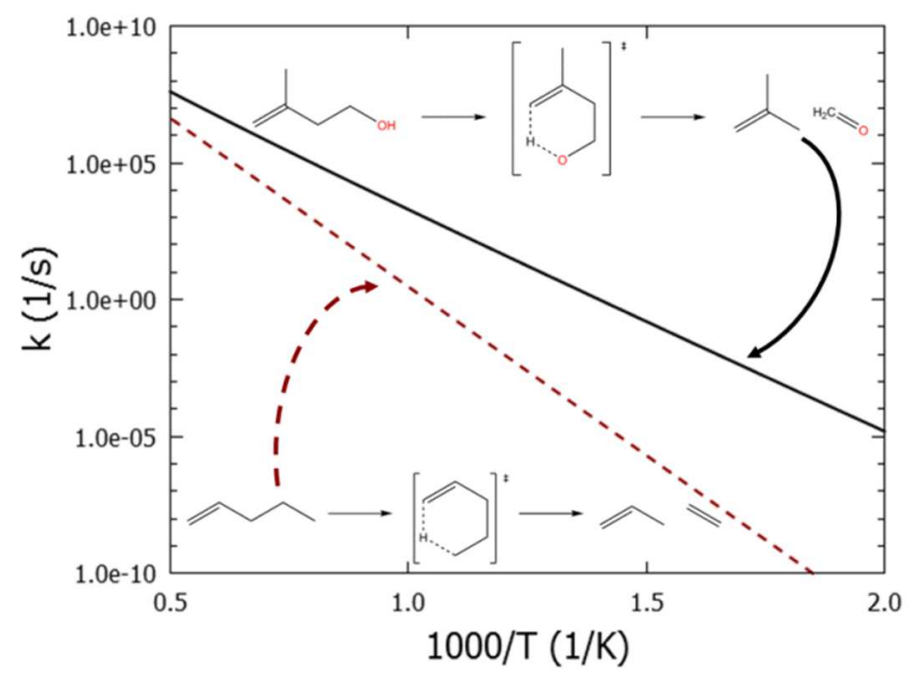

Figure 3: Comparison of the retroene-like decomposition reaction of isoprenol with the prototypical retro-ene reaction of 1-pentene, - isoprenol $\rightleftarrows$ isobutene + formaldehyde, --- 1 pentene $\rightleftarrows$ propene + ethene. 


\subsection{Thermochemistry of peroxy radicals}

Peroxy radicals are formed by reaction of molecular oxygen with carbon-centered radicals. In this work, the thermochemistry of peroxy prenol radicals has been calculated at the CBS-QB3 level of theory. The stability of peroxy radicals can be evaluated by calculating the bond dissociation energy (BDE) of the $\mathrm{C}-\mathrm{O}_{2}$ bond. The $\mathrm{C}-\mathrm{O}_{2} \mathrm{BDE}$ of several radicals are compared in Table 3.

Table 3: $\mathrm{C}-\mathrm{O}_{2}$ bond dissociation energies at $298 \mathrm{~K}$ for several peroxy radicals, calculated at the CBS-QB3 level of theory.

\begin{tabular}{|c|c|c|c|}
\hline Structure & $\mathrm{BDE} \mathrm{C}-\mathrm{O}_{2}\left(\mathrm{~kJ} \mathrm{~mol}^{-1}\right)$ & Structure & $\mathrm{BDE} \mathrm{C}-\mathrm{O}_{2}\left(\mathrm{~kJ} \mathrm{~mol}^{-1}\right)$ \\
\hline & 148 & & 166 \\
\hline & 77 & & 100 \\
\hline & 90 & & 95 \\
\hline & 90 & & 105 \\
\hline
\end{tabular}

Hydroxy-alkyl peroxy radicals have higher $\mathrm{C}^{-} \mathrm{O}_{2}$ BDE than alkyl peroxy radicals. The hydroxyl group has a stabilizing effect on the $\mathrm{C}_{-} \mathrm{O}_{2}$ bond through formation of a hydrogen bond. For example, ethyl-peroxy radicals have a $\mathrm{C}^{-} \mathrm{O}_{2} \mathrm{BDE}$ of $148 \mathrm{~kJ} \mathrm{~mol}^{-1}$ while 2-hydroxy-ethyl peroxy radicals have a $\mathrm{C}_{-} \mathrm{O}_{2} \mathrm{BDE}$ of $166 \mathrm{~kJ} \mathrm{~mol}^{-1}$, see Table 3. A hydrogen bond is formed between the hydroxyl hydrogen atom and either of the oxygen atoms of the peroxy group, e.g. the $\mathrm{C}(\mathrm{OO} \bullet) \mathrm{CCOH}$ radical contains a hydrogen bond with the first (carbon bounded) oxygen atom of the peroxy group (6-memebered ring), the $\mathrm{CC}(\mathrm{OO} \bullet) \mathrm{COH}$ radical contains a hydrogen bond with the second (terminal) oxygen atom of the peroxy group (6-memebered ring) and the $\mathrm{CCC}(\mathrm{OO} \bullet) \mathrm{OH}$ radical contains a hydrogen bond with the second (terminal) oxygen atom of the peroxy group (5-membered ring).

Allylic peroxy radicals, such as prenol peroxy radicals, have weaker $\mathrm{C}-\mathrm{O}_{2}$ bonds due to loss of resonance stabilization. The $\mathrm{C}-\mathrm{O}_{2} \mathrm{BDE}$ of several prenol peroxy radicals are compared with their isopentene analogs in Table 3. Similar to saturated alcohols, the presence of a hydroxyl group has a stabilizing effect on the $\mathrm{C}-\mathrm{O}_{2}$ bond in unsaturated alcohols. For example, the $\mathrm{C}-\mathrm{O}_{2}$ BDE of the 3-methyl-but-2-en-1-ol-1-peroxy radical is $100 \mathrm{~kJ} \mathrm{~mol}^{-1}, 23 \mathrm{~kJ} \mathrm{~mol}^{-1}$ higher than the $\mathrm{C}^{-} \mathrm{O}_{2} \mathrm{BDE}$ of the 3-methyl-but-2-en-1-peroxy radical. The effect of the hydroxyl group diminishes when the distance between the hydroxyl and peroxy group increases. The $\mathrm{C}_{-} \mathrm{O}_{2}$ BDE of trans- and cis-3-methyl-but-1-en-1-ol-3-peroxy radicals are 5 and $15 \mathrm{~kJ} \mathrm{~mol}^{-1}$ higher than the $\mathrm{C}^{-} \mathrm{O}_{2} \mathrm{BDE}$ of the 3-methyl-but-1-en-3-peroxy radical. 


\section{Results and discussion}

\subsection{Isoprenol}

\subsubsection{Experimental data}

Forty-three molecules were detected and quantified in the reactor effluent during isoprenol oxidation and pyrolysis. These include carbon oxides, hydrocarbons ranging from methane to phenanthrene and oxygenates containing a single oxygen atom. Mole fraction profiles for all detected species can be found in Supplementary Material.

The experimental isoprenol mole fraction profiles and the mole fraction profiles of the most representative products are presented in Figure 4 as a function of temperature, for $\varphi=0.5,1.0$ and $\infty$ (pyrolysis).

The isoprenol mole fraction profiles for all equivalence ratios are very similar. At the applied operating conditions, isoprenol starts to decompose at $625 \mathrm{~K}$ and conversion is approximately complete at $800 \mathrm{~K}$. The reactivity of isoprenol cannot be attributed to low-temperature oxidation chemistry as the conversion profile is independent of the presence of oxygen and as isoprenol pyrolysis and oxidation has a very high selectivity to isobutene and formaldehyde, the two products formed by molecular decomposition of isoprenol. No "negative temperature coefficient" (NTC) zone, a characteristic feature in the low-temperature oxidation of hydrocarbons and oxygenates with a long carbon chain, is observed.

In the case of isoprenol pyrolysis, the isobutene and formaldehyde mole fraction increases to approximately 0.008 , which corresponds to the initial isoprenol mole fraction in the feed, at 800 $\mathrm{K}$. The mole fractions of isobutene and formaldehyde in the reactor effluent remain relatively constant at 0.008 between $800 \mathrm{~K}$ and $950 \mathrm{~K}$. At higher temperatures, isobutene and formaldehyde mole fractions decrease, while mole fractions of other products, such as $\mathrm{CO}, \mathrm{CH}_{4}$, ethene, propene, propyne and allene increase.

In the case of isoprenol oxidation, the isobutene and formaldehyde mole fractions reach their maxima around $775 \mathrm{~K}$. At higher temperatures both mole fractions decrease and a wide variety of hydrocarbons and oxygenated molecules are formed, including 2-methyl-2-propenal, 2-methylpropanal and acetone. The latter molecules are known products of isobutene oxidation $[53,75]$ and motivates the inclusion of the low-temperature oxidation chemistry of this molecule into the current kinetic model.

\subsubsection{Kinetic model performance}

Reactor simulations, using the developed kinetic model, were conducted with the CHEMKIN PRO package using the perfectly stirred reactor module [76]. Calculated mole fraction profiles for isoprenol and products as a function of temperature have been added to Figure 4 for $\varphi=0.5,1.0$ and $\infty$. The predicted mole fraction profiles for isoprenol are independent of the equivalence ratio, which is in agreement with the experimental observation. Model calculated mole fraction profiles for isobutene and formaldehyde are in good agreement with the experimental data, i.e. a constant 
isobutene and formaldehyde mole fraction of approximately 0.008 between 800 and $950 \mathrm{~K}$ at $\varphi=$ $\infty$, while both molecules reach their maxima in mole fraction around $775 \mathrm{~K}$ at $\varphi=0.5$ and 1.0.
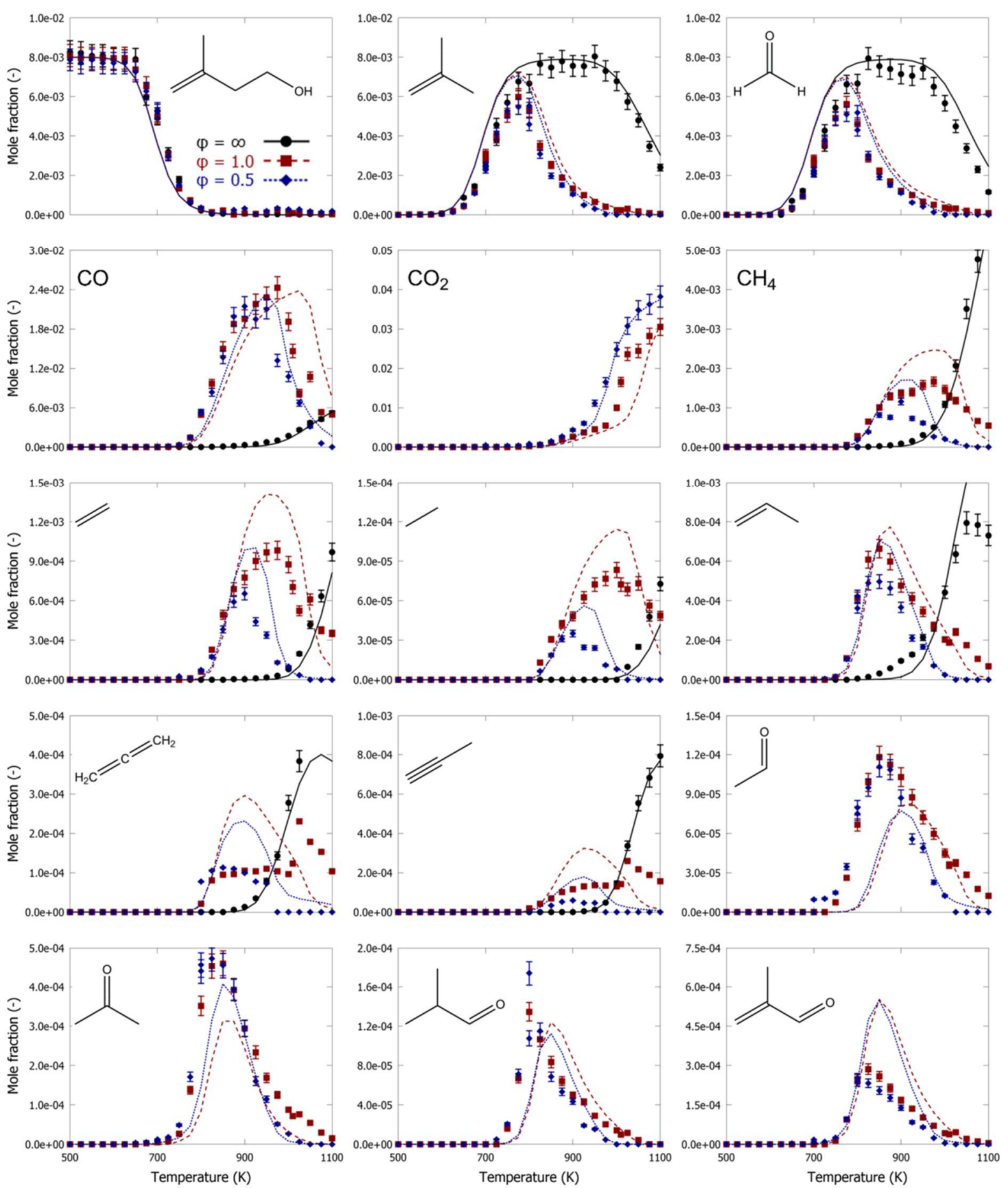

Figure 4: Mole fractions as a function of temperature for isoprenol oxidation and pyrolysis in a jet-stirred reactor, $\mathrm{P}=0.107 \mathrm{MPa}, \mathrm{F}_{\mathrm{V}}=4.0610^{-5} \mathrm{~m}^{3} \mathrm{~s}^{-1}, \tau=2 \mathrm{~s}, \mathrm{x}_{\text {isoprenol, }, 0}=0.008, \varphi=0.5$ (blue),$\varphi$

$=1.0$ (red) and $\varphi=\infty$ (black): symbols, experimental mole fraction profile of molecule represented in graph; lines, mole fraction profiles calculated with CHEMKIN using the perfectly stirred reactor model and the developed kinetic model. (For interpretation of the references to color in this figure legend, the reader is referred to the web version of this article.) 
The kinetic model accurately reproduces the effect of temperature and equivalence ratio on the mole fraction of the other products displayed in Figure 4. The main qualitative deviations of the kinetic model can be observed for propyne and allene, for which model calculated mole fraction profiles deviate from experimental mole fraction fractions at $\varphi=1.0$. Quantitative discrepancies are present for methane, ethene, acetaldehyde and 2-methyl-2-propenal.

Given the high selectivity towards isobutene, good model performance for isoprenol oxidation and pyrolysis requires an adequate isobutene submechanism. The used isobutene submechanism, see Section 4.1, has been tested against speciation data in a jet-stirred reactor [53] and flow reactor [77] by Zhou et al. [49]. Some discrepancies are observed in the study by Zhou et al. [49] and therefore it is not unsurprising that discrepancies are also observed in this work. Further validation of several submechanisms within the isobutene oxidation model by Zhou et al. [49], for example by obtaining experimental 2-methyl-2-propenal pyrolysis and oxidation speciation data, would be valuable.

\subsubsection{Reaction path analysis}

A reaction path analysis was performed and the results are displayed in Figure 5. Operating conditions correspond to $\varphi=1.0, \mathrm{x}_{\text {isoprenol }}=0.008, \mathrm{P}=0.107 \mathrm{MPa}, \tau=2.0 \mathrm{~s}, \mathrm{~T}=800 \mathrm{~K}$ and $1000 \mathrm{~K}$.

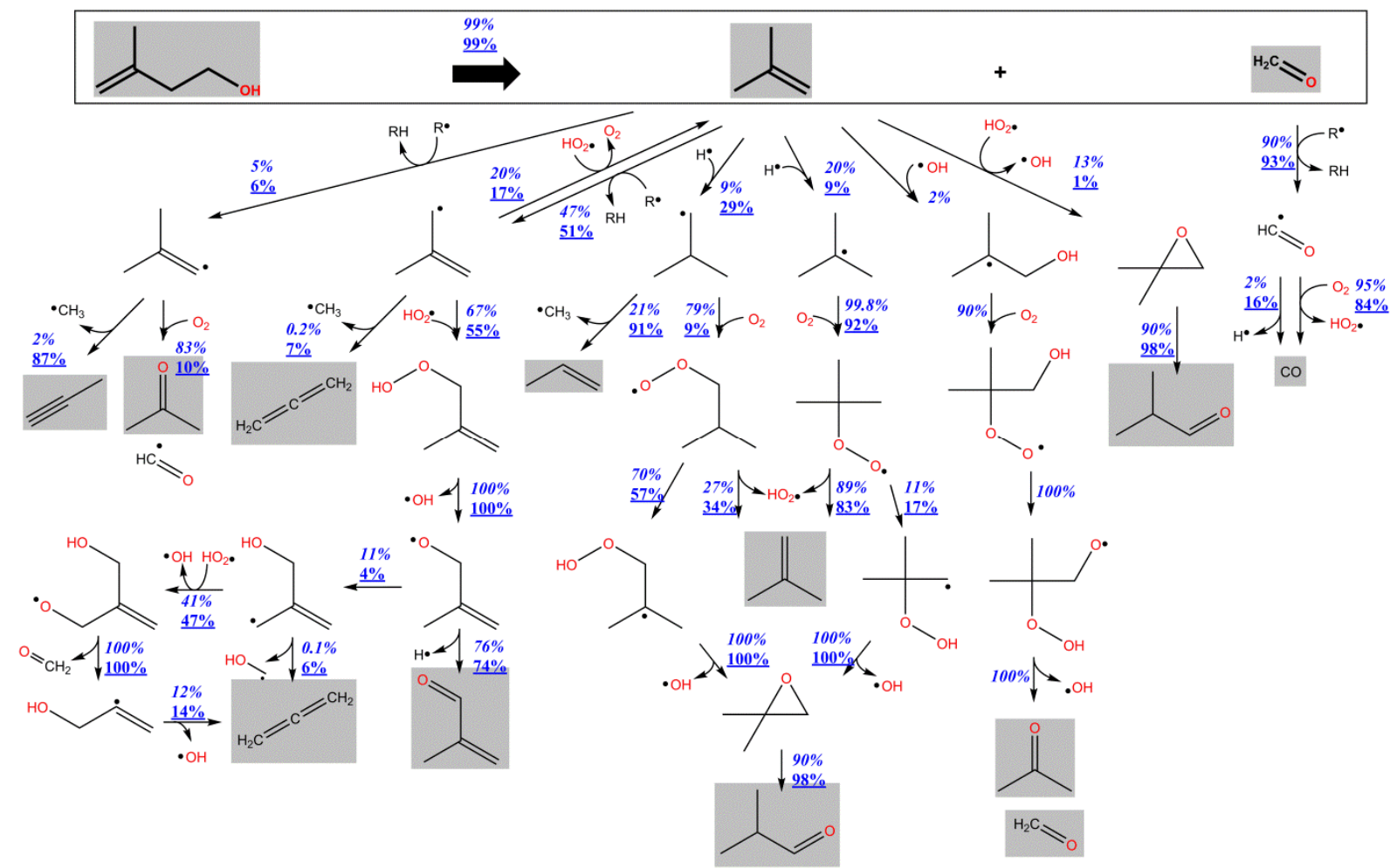

Figure 5: Reaction path analysis for the decomposition of isoprenol. Operating conditions: $\mathrm{F}_{\mathrm{V}}=$ $4.0610^{-5} \mathrm{~m}^{3} \mathrm{~s}^{-1}, \tau=2 \mathrm{~s}, \mathrm{x}_{\text {isoprenol, },}=0.008, \varphi=1.0, \mathrm{~T}=800 \mathrm{~K}$ (italic) and $1000 \mathrm{~K}$ (underlined).

Percentages on a reaction path represent the reaction rate relative to the total consumption rate of the reacting species. Species with a shaded background have been detected experimentally.

Isoprenol is almost exclusively consumed via unimolecular decomposition to isobutene and formaldehyde. This channel accounts for more than $99 \%$ of the total isoprenol consumption. 
Oxidation of isobutene leads to the majority of products detected in the reactor effluent. Hence, the performance of the kinetic model regarding the obtained experimental dataset depends on two key elements, i.e. correct prediction of unimolecular decomposition of isoprenol to isobutene and formaldehyde and the accuracy of the isobutene oxidation submechanism. The remainder of this paragraph focuses on the reaction paths leading to the major oxygenated intermediates displayed in Figure 4.

The major consumption path of isobutene is hydrogen abstraction from the allylic $\mathrm{C}-\mathrm{H}$ site forming the resonantly stabilized isobutenyl radical. Isobutenyl radicals preferentially recombine with hydroperoxy forming isobutenyl hydroperoxide. The $0-0$ bond in the latter molecule is weak and scission leads to isobutenyloxy and hydroxyl radicals. $\mathrm{C}-\mathrm{H} \beta$-scission of isobutenyloxy radical forms 2-methyl-2-propenal, one of the major intermediate oxygenated molecules detected experimentally. Isobutenyloxy radical can also isomerize forming a resonantly stabilized radical and subsequent decomposition is an important formation path of allene, see Figure 5. The aforementioned competing decomposition channels for isobutenyloxy radical are part of the base mechanism developed by Zhou et al. [49]. Associated reaction rate coefficients are estimated from the analogous allyloxy system [70] and rate rules. This may lead to erroneous branching ratio of the isobutenyloxy decomposition channels which can be the cause of the overprediction regarding 2-methyl-2-propenal and discrepancies regarding allene. At higher temperature and pyrolysis conditions, $\mathrm{C}-\mathrm{C} \beta$-scission of isobutenyl radical to allene and methyl is a non-negligible consumption channel of isobutenyl radicals.

Hydrogen abstraction from the vinylic carbon atom in isobutene is relatively slow compared to hydrogen abstraction from the allylic carbon atom. The product, 2-methyl-prop-1-en-1-yl radical, either reacts with molecular oxygen forming acetone and formyl radical, or, it produces propyne and methyl through C-C $\beta$-scission. Note that the calculated mole fraction profiles of propyne and allene deviate from experimental observations at oxidizing conditions. At low temperatures, both $\mathrm{C}_{3} \mathrm{H}_{4}$ species are over-predicted, and the observed sudden increase for $\varphi=1.0$ is not captured. Rate coefficients of several reactions related to propyne and allene formation were estimated from analogous reactive systems in the isobutene submechanism, e.g. rate coefficients for the reaction of 2-methyl-prop-1-en-1-yl radical with molecular oxygen were estimated from the reaction of vinyl with molecular oxygen [78]. The observed model performance suggests that additional theoretical calculations regarding radicals derived from isobutene and their interaction with oxygen would be beneficial.

Besides their involvement in hydrogen abstraction reactions, radicals can also add to the $\mathrm{C}=\mathrm{C}$ double bond of isobutene. Addition of hydroperoxy radical to isobutene leads to 2,2-dimethyloxirane and hydroxyl [53,75]. The major consumption path of 2,2-dimethyloxirane is unimolecular decomposition to 2-methyl-propanal. According to the developed model, addition of hydroperoxy radical to isobutene becomes insignificant at higher temperatures. Hydroxyl addition to the $\mathrm{C}=\mathrm{C}$ double bond is a minor consumption channel of isobutene. Molecular oxygen can add to the formed adduct. Intramolecular hydrogen abstraction of the hydroxyl-peroxy radical forming a hydroperoxy-alkoxy radical and subsequent $\beta$-scission leads to acetone, formaldehyde and hydroxyl. Addition of hydrogen atoms to isobutene can form tert-butyl and isobutyl radicals. $\beta$-scission reactions of butyl radicals are in competition with addition of molecular oxygen to butyl radicals forming peroxy radicals. At low temperatures, addition of hydrogen atoms on isobutene forming tert-butyl and subsequent addition of molecular oxygen dominates. At high temperatures, 
$\mathrm{C}-\mathrm{H} \beta$-scission of tert-butyl radical, back to isobutene, becomes more important. In contrast to tert-butyl radicals, isobutyl radicals can decompose by $\mathrm{C}-\mathrm{C} \beta$-scission, forming propene and methyl. The latter reaction path dominates over $\mathrm{C}-\mathrm{H} \beta$-scission of isobutyl, back to isobutene, and addition of molecular oxygen on isobutyl. Consumption of isobutene by addition of hydrogen atoms forming isobutyl radical is therefore larger than addition of hydrogen atoms forming tertbutyl radical at high temperatures.

Ethene and acetaldehyde are not presented in Figure 5 as they are not formed by primary consumption paths of isoprenol, isobutene and formaldehyde. Reaction path analysis reveals that ethene is mostly formed by (i) hydrogen atom addition to propene to 1-propyl followed by C-C $\beta$-scission and (ii) isomerization of allyloxy to 3-oxo-1-propyl followed by $C-C \beta$-scission. Reaction path analysis reveals that acetaldehyde is mostly formed by (i) $\mathrm{C}-\mathrm{H} \beta$-scission of ethoxy radical, (ii) hydroxyl radical addition to propene forming 2-hydroxy-1-propyl followed by addition of molecular oxygen and decomposition by a Waddington mechanism to acetaldehyde, formaldehyde and hydroxyl radical and (iii) oxidation of the propen-2-yl radical. Model performance for these species may improve if the assigned rate coefficients are updated by theoretical investigation of these reactions and the competing reaction pathways, but this is outside the scope of this study.

\subsection{Prenol}

\subsubsection{Experimental data}

Forty-two molecules were detected and quantified in the reactor effluent during prenol oxidation and pyrolysis. Mole fraction profiles for all detected species can be found in Supplementary material.

The experimental prenol mole fraction profiles and the mole fraction profiles of the most representative products are presented in Figure 6 as a function of temperature, for $\varphi=0.5,1.0$ and $\infty$.

In contrast to isoprenol, the presence of oxygen in the reactor inlet has a significant influence on the prenol conversion. At $\varphi=0.5$ and 1.0, prenol conversion starts at approximately $650 \mathrm{~K}$ and is almost complete at $900 \mathrm{~K}$. At $\varphi=\infty$, prenol conversion starts at approximately $850 \mathrm{~K}$ and is almost complete at $1050 \mathrm{~K}$. No clear NTC-zone is observed, but several product mole fraction evolutions (e.g. 3-methyl-2-butenal, acetone, 2-methyl-propanal) display two maxima, one below and one above $750 \mathrm{~K}$.

Prenol oxidation and pyrolysis have high selectivity's to 3-methy-2-butenal and 2-methyl-1,3butadiene at the conditions investigated. 3-methyl-2-butenal has a higher yield at $\varphi=0.5$ and 1.0 than at $\varphi=\infty$. This is consistent with other comparable study's regarding alcohols $[1,79]$. The reaction of the $\alpha$-hydroxyalkyl radical with molecular oxygen is a known important reaction path to aldehydes in the oxidation of alcohols $[1,73]$. Obviously, this reaction path is of no significance in the pyrolysis of alcohols. 

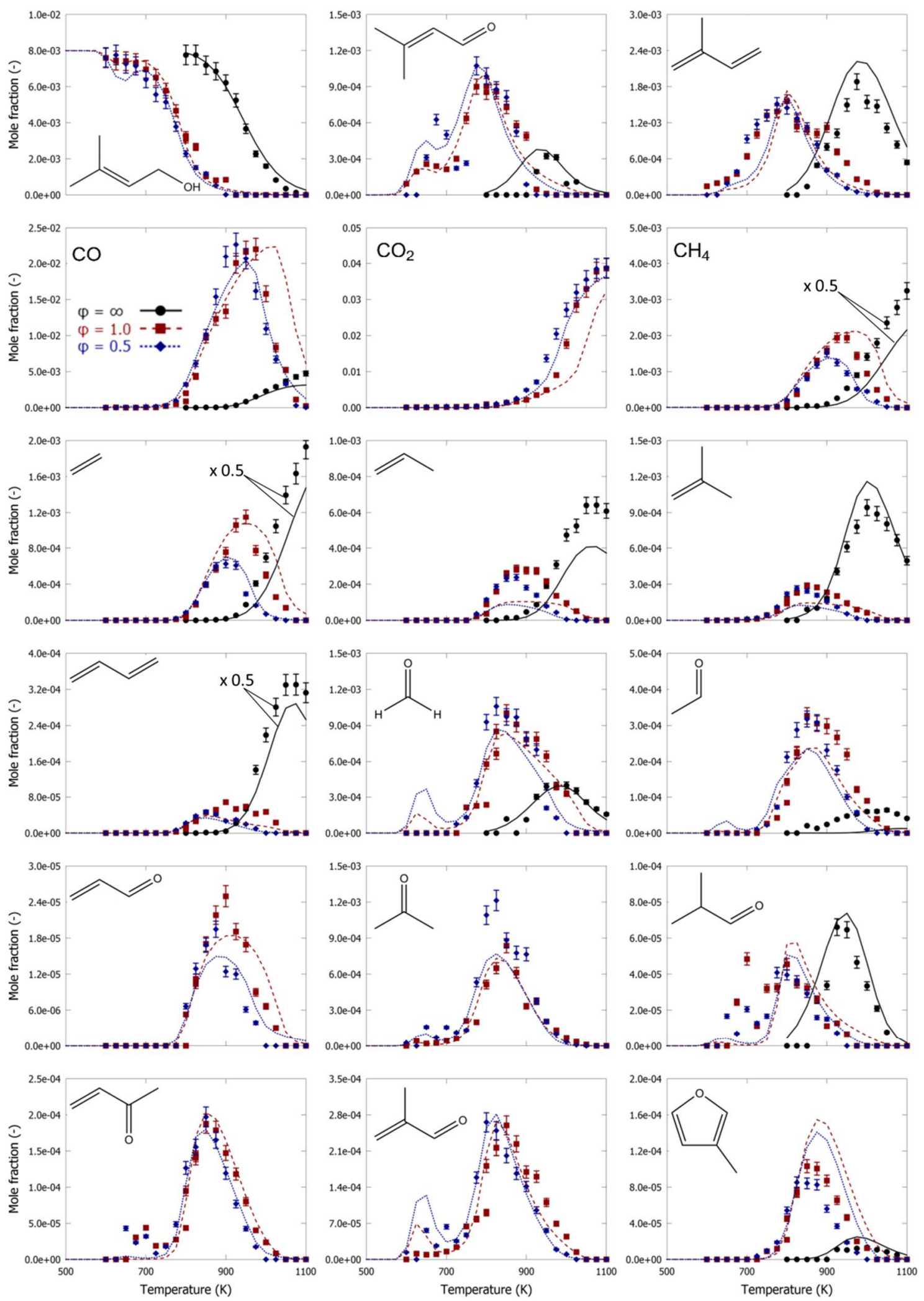

Figure 6: Mole fractions as a function of temperature for prenol oxidation and pyrolysis in a jetstirred reactor, $\mathrm{P}=0.107 \mathrm{MPa}, \mathrm{F}_{\mathrm{V}}=4.0610^{-5} \mathrm{~m}^{3} \mathrm{~s}^{-1}, \tau=2 \mathrm{~s}, \mathrm{x}_{\text {prenol }, 0}=0.008, \varphi=0.5$ (blue), $\varphi=1.0$ (red) and $\varphi=\infty$ (black): symbols, experimental mole fraction profile of molecule represented in graph; lines, mole fraction profiles calculated with CHEMKIN using the perfectly stirred reactor model and the developed kinetic model. (For interpretation of the references to color in this figure legend, the reader is referred to the web version of this article.) 
Besides hydrocarbons and small oxygenated molecules, the reactor effluent includes acetone, 2-methyl-2-propenal and 3-buten-2-one. A recent kinetic modeling study regarding the oxidation of 2-methyl-2-butene, which is the hydrocarbon backbone of prenol, highlighted the importance of hydroperoxy recombination with allylic radicals [69]. Reaction path analysis showed that the latter reactions, and subsequent decomposition, can lead to 2-methyl-2-propenal and 3-buten-2one. Hydroxyl radical addition on 2-methyl-2-butene and reaction with molecular oxygen, the Waddington mechanism, leads to acetaldehyde, acetone and hydroxyl radical [69].

\subsubsection{Kinetic model performance}

Reactor simulations, using the developed kinetic model, were conducted with the CHEMKIN PRO package using the perfectly stirred reactor module [76]. Model calculated mole fraction profiles for prenol and products as a function of temperature have been added to Figure 6 for $\varphi=0.5,1.0$ and $\infty$.

The calculated prenol mole fraction profiles are generally in good agreement with the experimental data, i.e. the effect of temperature and equivalence ratio is accurately reproduced by the kinetic model. Prenol conversion is slightly overestimated below $700 \mathrm{~K}$ for $\varphi=0.5$. This predicted minor NTC behavior is not observed in the experimental prenol mole fraction profile.

The 3-methyl-2-butenal profiles, including the local maxima below $700 \mathrm{~K}$, are well reproduced by the kinetic model. The predicted 2-methyl-1,3-butadiene mole fraction profiles matches the experimental mole fraction profile at pyrolysis conditions, while in the presence of oxygen, the kinetic model underestimates 2-methyl-1,3-butadiene yields below $750 \mathrm{~K}$ and above $850 \mathrm{~K}$. The calculated mole fraction profiles of other products are in reasonable agreement with the experimental data. The kinetic model underpredicts propene mole fraction profiles. Discrepancies below $750 \mathrm{~K}$ can be observed for formaldehyde, 2-methyl-propanal and 3-oxo-1-butene.

\subsubsection{Reaction path analysis}

A reaction path analysis was performed to highlight important prenol consumption routes and to identify critical product formation channels. Operating conditions correspond to $\varphi=1.0, \mathrm{x}_{\text {prenol }}=$ $0.008, \mathrm{P}=0.107 \mathrm{MPa}, \tau=2.0 \mathrm{~s}, \mathrm{~T}=700 \mathrm{~K}$ and $900 \mathrm{~K}$. Prenol conversion is $6 \%$ at $700 \mathrm{~K}$ and $96 \%$ at $900 \mathrm{~K}$ respectively. The results are displayed in Figure 7.

\subsubsection{Prenol decomposition paths}

Prenol consumption is dominated by hydrogen abstraction. Hydroxyl is the main hydrogen abstracting radical in oxidation conditions. Hydrogen abstraction from the $\alpha$ - and $\delta$-carbon atoms leads to resonantly stabilized radicals. The $\alpha$-prenol radicals can react with molecular oxygen forming 3-methyl-2-butenal and hydroperoxy radical (reactions 49 and 50 in Table 1). In their study regarding the chlorine atom-initiated low-temperature oxidation of prenol, Welz et al. highlighted that this is a low-energy reaction path [29]. At pyrolysis conditions, 3-methyl-2butenal is formed by direct $\mathrm{O}-\mathrm{H} \beta$-scission of $\alpha$-prenol radicals (reaction 31 in Table 1 ).

Hydrogen abstraction from the $\delta$-carbon atom is the main prenol consumption channel. Intramolecular hydrogen abstraction of the $\delta$-prenol radical forms the $\alpha$-prenol radical, and vice 
versa, which proceeds through a 5-membered transition state (reaction 34 in Table 1). At the operating conditions of Figure 7 , intramolecular hydrogen abstraction from $\delta$-prenol radical to $\alpha$-prenol radical accounts for approximately $18 \%$ and $5 \%$ of the total $\delta$-prenol radical consumption rate at $700 \mathrm{~K}$ and $900 \mathrm{~K}$, respectively. $\beta$-scission of the $\mathrm{C}-\mathrm{O}$ bond is the dominant $\delta$-prenol radical decomposition channel and the main formation route of 2-methyl-1,3-butadiene (reaction 33 in Table 1).

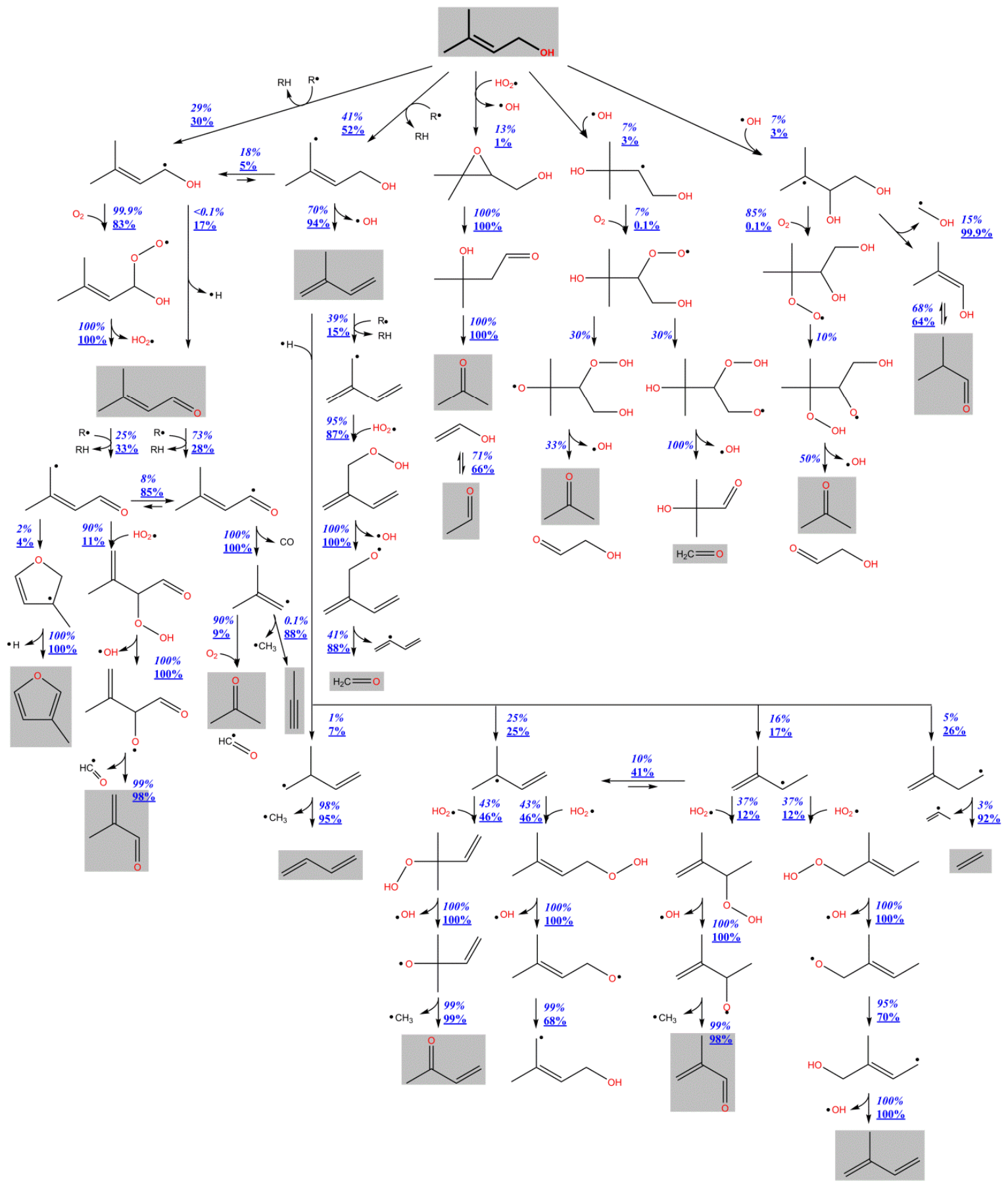

Figure 7: Reaction path analysis for the decomposition of prenol. Operating conditions: $\mathrm{F}_{\mathrm{V}}=4.06$ $10^{-5} \mathrm{~m}^{3} \mathrm{~s}^{-1}, \tau=2 \mathrm{~s}, \mathrm{x}_{\text {prenol, }, 0}=0.008, \varphi=1.0, \mathrm{~T}=700 \mathrm{~K}$ (blue and italic) and $900 \mathrm{~K}$ (red and underlined). Percentages on a reaction path represent the reaction rate relative to the total consumption rate of the reacting species. Species with a shaded background have been detected experimentally. (For interpretation of the references to color in this figure legend, the reader is referred to the web version of this article.) 
Hydroperoxy radical can add to the $\mathrm{C}=\mathrm{C}$ double bond forming 3,3-dimethyl-2-methanol-oxiran and hydroxyl radical. This type of reaction is known to be important in the oxidation of alkenes as it consumes a relatively unreactive hydroperoxy radical and produces a reactive hydroxyl radical [19]. 3,3-dimethyl-2-methanol-oxiran isomerizes to 3-methyl-3-hydroxy-butanal following hydrogen migration from the hydroxyl group to the ring oxygen atom (calculated at the CBS-QB3 level of theory $-\mathrm{k}\left[\mathrm{s}^{-1}\right]=2.78 \cdot 10^{10} \mathrm{~T}^{0.526} \mathrm{e}^{-25,226 / \mathrm{T}}$ ). 3-methyl-3-hydroxy-butanal can react by a retro-ene reaction to acetone and ethenol. Addition of hydroperoxy radicals is a minor prenol consumption path at higher temperatures. Note that oxygenated molecules with both a hydroxyl and an oxo-functionality, such as 3-methyl-3-hydroxy-butanal and 2-hydroxy-acetaldehyde, were not detected in the reactor effluent.

Hydroxyl radical addition to the $\mathrm{C}=\mathrm{C}$ double bond (reactions 34 and 42 in Table 1), followed by addition of molecular oxygen leads to hydroxylated peroxy radicals (reactions 37 and 44 in Table 1), which can isomerize to hydroperoxy-alkoxy radicals by intermolecular hydrogen abstraction (reactions 38, 40 and 45 in Table 1). These radicals likely decompose by $\beta$-scission of the C-C bond, forming a hydroxyl radical and two carbonyl-containing molecules, the Waddington mechanism.

Hydrogen atom addition to the $\mathrm{C}=\mathrm{C}$ double bond accounts for approximately $10 \%$ of the total prenol consumption at $900 \mathrm{~K}$. The resulting pentanol radicals mainly decompose by $\beta$-scission reactions, i.e. 3-methyl-1-hydroxy-2-butyl to hydroxyl and 3-methyl-1-butene and 2-methyl-4hydroxy-2-butyl to isobutene and hydroxyl-methyl. The latter reaction path is one of the main isobutene formation paths. Note that the mole fraction profiles of isobutene and propene, which is a primary product of isobutene oxidation and pyrolysis (see Figure 5), are underpredicted by the kinetic model. An adjustment of the rate coefficient for the hydrogen atom addition pathway might improve model performance but such optimizations are not an objective of this study.

\subsubsection{Decomposition of primary products}

The reaction path analysis reveals that a large fraction of the product spectrum formed during the oxidation and pyrolysis of prenol originates from the consumption of 3-methyl-2-butenal and 2-methyl-1,3-butadiene.

Similar to prenol, 3-methyl-2-butenal mainly reacts by hydrogen abstraction forming resonantly stabilized radicals, 3-methyl-but-2-en-1-oxo-1-yl and 3-methyl-but-3-en-1-oxo-2-yl, see Figure 7. Both radicals can interconvert by intramolecular hydrogen abstraction.

CO $\alpha$-scission of 3-methyl-but-2-en-1-oxo-1-yl radical forms 2-methyl-prop-1-en-1-yl radical. This vinylic radical can react by $\mathrm{C}-\mathrm{C} \beta$-scission forming propyne and methyl or it produces acetone and formyl after reacting with molecular oxygen. The latter reaction path is the main acetone formation path. As can be observed from Figure 6, the effect of equivalence ratio on the acetone mole fraction is more pronounced in the experimental profile than the model calculated profile. Acetone formation paths that involve oxygen, such as reaction of 2-methyl-prop-1-en-1-yl with molecular oxygen, warrant further investigation to resolve the observed discrepancies.

3-methyl-but-3-en-1-oxo-2-yl can recombine with a hydroperoxy radical. Scission of the 0-0 bond and subsequent decomposition may form 2-methyl-2-propenal and a formyl radical. 
Alternatively, 3-methyl-but-3-en-1-oxo-2-yl radical can react by intramolecular radical addition forming a 5-membered ring. $\mathrm{C}-\mathrm{H} \beta$-scission of the cyclic radical forms 3 -methyl-furan, which was experimentally observed in this work and in the oxidation study by Welz et al. [29].

Besides 3-methyl-2-butenal, 2-methyl-1,3-butadiene is an important product in the oxidation and pyrolysis of prenol. One possible reaction route is hydrogen abstraction from the allylic carbon atom, $39 \%$ and $15 \%$ of the total 2-methyl-1,3-butadiene consumption rate at $700 \mathrm{~K}$ and $900 \mathrm{~K}$ respectively. $\mathrm{C}-\mathrm{C} \beta$-scission of the resulting radical is slow at the investigated operating conditions. Instead, the radical will mainly abstract hydrogen from other species, reforming 2-methyl-1,3-butadiene, or recombine with a hydroperoxy radical. The main consumption path of 2-methyl-1,3-butadiene is addition of hydrogen atoms to one of its double bonds, $47 \%$ and $75 \%$ of the total 2-methyl-1,3-butadiene consumption rate at $700 \mathrm{~K}$ and $900 \mathrm{~K}$ respectively. Hydrogen addition forming resonantly stabilized radicals, 3-methyl-but-2-en-1-yl and 2-methyl-but-2-en-1$\mathrm{yl}$, is favored. Both radicals can interconvert by intramolecular hydrogen abstraction. Similar to some of the other resonantly stabilized radicals encountered in this work, recombination with hydroperoxy radicals is the major reaction channel. This is in agreement with the conclusions by Welz et al. for 2-methyl-2-butene oxidation [69]. Hydrogen addition to 2-methyl-1,3-butadiene can also form non-resonantly stabilized radicals, i.e. 2-methyl-but-3-en-1-yl and 3-methyl-but-3en-1-yl. These radicals mainly react by $C-C \beta$ scission forming 1,3-butadiene and methyl and by $\mathrm{C}-\mathrm{C} \beta$ scission forming ethene and propen-2-yl radical, respectively.

\subsubsection{Low-temperature reactivity}

Characteristic for the oxidation of prenol is the occurrence of aldehyde peaks below $700 \mathrm{~K}$. Such early formation of oxygenated products has not been observed during the oxidation of 2-methyl2-butene [69], the hydrocarbon backbone of prenol.

According to the model, this difference can be explained by stabilization effects of the hydroxyl group on peroxy radicals, as has been discussed earlier. The higher stability enables intramolecular hydrogen abstractions which can be followed by fragmentation to aldehydes and/or chain branching reactions.

Reaction path analysis reveals that addition of molecular oxygen to the $\delta$-position is responsible for the chain branching, necessary to trigger low-temperature chemistry. Intramolecular hydrogen abstraction of the peroxy radical through a 7-membered cyclic transition state and subsequent reaction with molecular oxygen will form 4-hydroperoxy-3-methyl-2-butenal (reactions 50, 51 and 52 in Table 1), see Figure 8. The latter hydroperoxide reacts by $0-0$ scission.

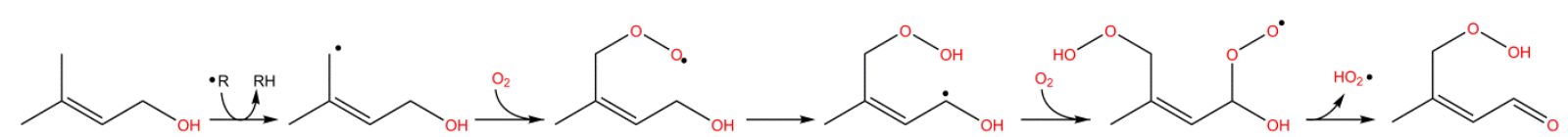

Figure 8: Chain branching reaction path in the oxidation of prenol.

Addition of molecular oxygen to $\delta$-prenol radicals is in competition with addition of molecular oxygen to $\alpha$-prenol radicals. The latter reaction path leads to 3-methyl-2-butenal and a hydroperoxy radical and reduces reactivity. 
Due to the strong non-nearest neighbor stabilization effects observed in hydroxylated peroxy radicals, group-additivity is not an accurate means to provide the thermochemistry. Similarly, rate coefficients deduced from reactions of reactants that do not contain the hydroxyl functionality have high uncertainties. Improved predictions of the developed kinetic model thus require a systematic quantum-mechanical study of all important reactions. This is beyond the scope of the current study and subject to future work.

\section{Conclusions}

The oxidation and pyrolysis of two unsaturated alcohols, prenol and isoprenol, have been studied in the temperature range of 500-1100 K. It is shown that they react entirely different and that a newly constructed kinetic model reproduces these reactivity differences well.

Isoprenol oxidation and pyrolysis is dominated by a single unimolecular reaction giving formaldehyde and isobutene. The reaction proceeds through a 6-membered transition state and is likely to be important for all unsaturated alcohols which contain the $\mathrm{C}=\mathrm{C}$ double bond in $\gamma$-position relative to the hydroxyl group. Other molecules in the reactor effluent are formed by oxidation and pyrolysis of isobutene and formaldehyde.

The prenol results show that the different position of the double bond completely changes the reactivity, i.e. from molecular channels to radical chemistry. Though prenol reacts through typical pathways, such as hydrogen abstraction and radical addition on the $\mathrm{C}=\mathrm{C}$ double bond, the presence of a hydroxyl functional group has a clear impact on the thermochemistry. This enables minor low-temperature chemistry in prenol oxidation, resulting in the formation of aldehydes and ketones. Low-temperature chemistry is not observed during the oxidation of 2-methyl-2-butene, which has the same hydrocarbon structure as prenol.

\section{Acknowledgments}

The authors acknowledge the financial support from the Long Term Structural Methusalem Funding by the Flemish Government, the European Research Council under the European Union's Seventh Framework Programme (FP7/2007-2013)/ERC grant agreement no. 290793, the Research Board of Ghent University (BOF) and the Fund for Scientific Research Flanders (FWO). The SBO proposal "Bioleum" supported by the Institute for promotion of Innovation through Science and Technology in Flanders (IWT) is acknowledged. Computational support was provided by the STEVIN Supercomputer Infrastructure at Ghent University, funded by Ghent University, the Flemish Supercomputer Center (VSC), the Hercules Foundation and the Flemish Government Department EWI.

\section{Supplementary Materials}

- $\quad$ Experimental data for oxidation and pyrolysis of isoprenol and prenol

- $\quad$ Reaction families Genesys

- $\quad$ Kinetic model 


\section{References}

[1] S.M. Sarathy, P. Oßwald, N. Hansen, K. Kohse-Höinghaus, Alcohol combustion chemistry, Prog. Energy Combust. Sci., 44 (2014), 40-102

[2] K. Kohse-Hoinghaus, P. Osswald, T.A. Cool, T. Kasper, N. Hansen, F. Qi, C.K. Westbrook, P.R. Westmoreland, Biofuel combustion chemistry: from ethanol to biodiesel, Angew. Chem. Int. Ed., 49 (2010), 3572-3597

[3] G. Wang, W. Yuan, Y. Li, L. Zhao, F. Qi, Experimental and kinetic modeling study of n-pentanol pyrolysis and combustion, Combust. Flame, 162 (2015), 3277-3287

[4] X.Y. Zhang, B. Yang, W.H. Yuan, Z.J. Cheng, L.D. Zhang, Y.Y. Li, F. Qi, Pyrolysis of 2-methyl-1butanol at low and atmospheric pressures: Mass spectrometry and modeling studies, Proc. Combust. Inst., 35 (2015), 409-417

[5] L. Cai, Y. Uygun, C. Togbé, H. Pitsch, H. Olivier, P. Dagaut, S.M. Sarathy, An experimental and modeling study of n-octanol combustion, Proc. Combust. Inst., 35 (2015), 419-427

[6] A. Lucassen, S. Park, N. Hansen, S.M. Sarathy, Combustion chemistry of alcohols: Experimental and modeled structure of a premixed 2-methylbutanol flame, Proc. Combust. Inst., 35 (2015), 813-820

[7] S. Park, O. Mannaa, F. Khaled, R. Bougacha, M.S. Mansour, A. Farooq, S.H. Chung, S.M. Sarathy, A comprehensive experimental and modeling study of 2-methylbutanol combustion, Combust. Flame, 162 (2015), 2166-2176

[8] Z. Serinyel, C. Togbé, G. Dayma, P. Dagaut, An experimental and modeling study of 2-methyl-1butanol oxidation in a jet-stirred reactor, Combust. Flame, 161 (2014), 3003-3013

[9] Y. Zheng, Q. Liu, L. Li, W. Qin, J. Yang, H. Zhang, X. Jiang, T. Cheng, W. Liu, X. Xu, M. Xian, Metabolic engineering of Escherichia coli for high-specificity production of isoprenol and prenol as next generation of biofuels, Biotechnol. Biofuels, 6 (2013), p. 57

[10] K.W. George, M.G. Thompson, A. Kang, E. Baidoo, G. Wang, L.J.G. Chan, P.D. Adams, C.J. Petzold, J.D. Keasling, T.S. Lee, Metabolic engineering for the high-yield production of isoprenoid-based C5 alcohols in E-coli, Sci. Rep., 5 (2015)

[11] K.A. Heufer, J. Bugler, H.J. Curran, A comparison of longer alkane and alcohol ignition including new experimental results for n-pentanol and n-hexanol, Proc. Combust. Inst., 34 (2013), 511-518

[12] K.A. Heufer, S.M. Sarathy, H.J. Curran, A.C. Davis, C.K. Westbrook, W.J. Pitz, Detailed kinetic modeling study of n-pentanol oxidation, Energy Fuel., 26 (2012), 6678-6685

[13] S. Mani Sarathy, S. Park, B.W. Weber, W. Wang, P.S. Veloo, A.C. Davis, C. Togbe, C.K. Westbrook, O. Park, G. Dayma, Z. Luo, M.A. Oehlschlaeger, F.N. Egolfopoulos, T. Lu, W.J. Pitz, C.-J. Sung, P. 
Dagaut, A comprehensive experimental and modeling study of iso-pentanol combustion, Combust. Flame, 160 (2013), 2712-2728

[14] G. da Silva, J.W. Bozzelli, L. Liang, J.T. Farrell, Ethanol oxidation: kinetics of the $\alpha$-hydroxyethyl radical + 02 reaction, J. Phys. Chem. A, 113 (2009), 8923-8933

[15] O. Welz, J. Zádor, J.D. Savee, L. Sheps, D.L. Osborn, C.A. Taatjes, Low-temperature combustion chemistry of n-butanol: principal oxidation pathways of hydroxybutyl radicals, J. Phys. Chem. A, 117 (2013), 11983-12001

[16] O. Welz, J. Zador, J.D. Savee, M.Y. Ng, G. Meloni, R.X. Fernandes, L. Sheps, B.A. Simmons, T.S. Lee, D.L. Osborn, C.A. Taatjes, Low-temperature combustion chemistry of biofuels: pathways in the initial low-temperature ( $550 \mathrm{~K}-750 \mathrm{~K}$ ) oxidation chemistry of isopentanol, Phys. Chem. Chem. Phys., 14 (2012), 3112-3127

[17] A. Fridlyand, S.S. Goldsborough, K. Brezinsky, S.S. Merchant, W.H. Green, Influence of the double bond position on the oxidation of decene isomers at high pressures and temperatures, Proc. Combust. Inst., 35 (2015), 333-340

[18] M. Mehl, G. Vanhove, W.J. Pitz, E. Ranzi, Oxidation and combustion of the n-hexene isomers: a wide range kinetic modeling study, Combust. Flame, 155 (2008), 756-772

[19] F. Battin-Leclerc, A. Rodriguez, B. Husson, O. Herbinet, P.-A. Glaude, Z. Wang, Z. Cheng, F. Qi, Products from the oxidation of linear isomers of hexane, J. Phys. Chem. A, 118 (2014), 673-683

[20] R. Bounaceur, V. Warth, B. Sirjean, P.A. Glaude, R. Fournet, F. Battin-Leclerc, Influence of the position of the double bond on the autoignition of linear alkenes at low temperature, Proc. Combust. Inst., 32 (2009), 387-394

[21] C.A. Taatjes, N. Hansen, J.A. Miller, T.A. Cool, J. Wang, P.R. Westmoreland, M.E. Law, T. Kasper, K. Kohse-Höinghaus, Combustion chemistry of enols: possible ethenol precursors in flames, J. Phys. Chem. A, 110 (2006), 3254-3260

[22] C.A. Taatjes, N. Hansen, A. McIlroy, J.A. Miller, J.P. Senosiain, S.J. Klippenstein, F. Qi, L. Sheng, Y. Zhang, T.A. Cool, J. Wang, P.R. Westmoreland, M.E. Law, T. Kasper, K. Kohse-Höinghaus, Enols are common intermediates in hydrocarbon oxidation, Science, 308 (2005), 1887-1889

[23] J.P. Senosiain, S.J. Klippenstein, J.A. Miller, Reaction of ethylene with hydroxyl radicals: a theoretical study, J. Phys. Chem. A, 110 (2006), 6960-6970

[24] J. Zador, A.W. Jasper, J.A. Miller, The reaction between propene and hydroxyl, Phys. Chem. Chem. Phys., 11 (2009), 11040-11053

[25] L.K. Huynh, H.R. Zhang, S. Zhang, E. Eddings, A. Sarofim, M.E. Law, P.R. Westmoreland, T.N. Truong, Kinetics of enol formation from reaction of $\mathrm{OH}$ with propene, J. Phys. Chem. A, 113 (2009), 3177-3185 
[26] G. da Silva, J.W. Bozzelli, Role of the $\alpha$-hydroxyethylperoxy radical in the reactions of acetaldehyde and vinyl alcohol with HO2, Chem. Phys. Lett., 483 (2009), 25-29

[27] G. da Silva, Carboxylic acid catalyzed keto-enol tautomerizations in the gas phase, Angew. Chem. Int. Ed., 49 (2010), 7523-7525

[28] A. Rodríguez, D. Rodríguez, A. Soto, I. Bravo, Y. Diaz-de-Mera, A. Notario, A. Aranda, Products and mechanism of the reaction of $\mathrm{Cl}$ atoms with unsaturated alcohols, Atmos. Environ., 50 (2012), $214-224$

[29] O. Welz, J.D. Savee, D.L. Osborn, C.A. Taatjes, Chlorine atom-initiated low-temperature oxidation of prenol and isoprenol: the effect of CC double bonds on the peroxy radical chemistry in alcohol oxidation, Proc. Combust. Inst., 35 (2015), 401-408

[30] C.K. Westbrook, W.J. Pitz, S.M. Sarathy, M. Mehl, Detailed chemical kinetic modeling of the effects of CC double bonds on the ignition of biodiesel fuels, Proc. Combust. Inst., 34 (2013), 30493056

[31] N.M. Vandewiele, K.M. Van Geem, M.-F. Reyniers, G.B. Marin, Genesys: kinetic model construction using chemo-informatics, Chem. Eng. J., 207 (2012), 526-538

[32] 0. Herbinet, F. Battin-Leclerc, Progress in understanding low-temperature organic compound oxidation using a jet-stirred reactor, Int. J. Chem. Kinet., 46 (2014), 619-639

[33] O. Herbinet, B. Husson, Z. Serinyel, M. Cord, V. Warth, R. Fournet, P.-A. Glaude, B. Sirjean, F. Battin-Leclerc, Z. Wang, M. Xie, Z. Cheng, F. Qi, Experimental and modeling investigation of the low-temperature oxidation of n-heptane, Combust. Flame, 159 (2012), 3455-3471

[34] O. Herbinet, P.M. Marquaire, F. Battin-Leclerc, R. Fournet, Thermal decomposition of ndodecane: experiments and kinetic modeling, J. Anal. Appl. Pyrolysis, 78 (2007), 419-429

[35] Gaussian 09, Revision B.01, M.J. Frisch, G.W. Trucks, H.B. Schlegel, G.E. Scuseria, M.A. Robb, J.R. Cheeseman, G. Scalmani, V. Barone, B. Mennucci, G.A. Petersson, H. Nakatsuji, M. Caricato, X. Li, H.P. Hratchian, A.F. Izmaylov, J. Bloino, G. Zheng, J.L. Sonnenberg, M. Hada, M. Ehara, K. Toyota, R. Fukuda, J. Hasegawa, M. Ishida, T. Nakajima, Y. Honda, O. Kitao, H. Nakai, T. Vreven, J.A. Montgomery, J.E. Peralta, F. Ogliaro, M. Bearpark, J.J. Heyd, E. Brothers, K.N. Kudin, V.N. Staroverov, R. Kobayashi, J. Normand, K. Raghavachari, A. Rendell, J.C. Burant, S.S. Iyengar, J. Tomasi, M. Cossi, N. Rega, J.M. Millam, M. Klene, J.E. Knox, J.B. Cross, V. Bakken, C. Adamo, J. Jaramillo, R. Gomperts, R.E. Stratmann, O. Yazyev, A.J. Austin, R. Cammi, C. Pomelli, J.W. Ochterski, R.L. Martin, K. Morokuma, V.G. Zakrzewski, G.A. Voth, P. Salvador, J.J. Dannenberg, S. Dapprich, A.D. Daniels, Farkas, J.B. Foresman, J.V. Ortiz, J. Cioslowski, D.J. Fox, Gaussian, Inc., Wallingford, CT, 2009.

[36] J.A. Montgomery Jr, M.J. Frisch, A complete basis set model chemistry. VI. Use of density functional geometries and frequencies, J. Chem. Phys., 110 (1999), 2822

[37] A.L.L. East, L. Radom, An initio statistical thermodynamical models for the computation of third-law entropies, J. Chem. Phys., 106 (1997), 6655 
[38] L.A. Curtiss, K. Raghavachari, P.C. Redfern, J.A. Pople, Assessment of Gaussian-2 and density functional theories for the computation of enthalpies of formation, J. Chem. Phys., 106 (1997), 1063-1079

[39] G.A. Petersson, D.K. Malick, W.G. Wilson, J.W. Ochterski, J.A. Montgomery, M.J. Frisch, Calibration and comparison of the Gaussian-2, complete basis set, and density functional methods for computational thermochemistry, J. Chem. Phys., 109 (1998), 10570-10579

[40] L.A. Curtiss, K. Raghavachari, P.C. Redfern, V. Rassolov, J.A. Pople, Gaussian-3 (G3) theory for molecules containing first and second-row atoms, J. Chem. Phys., 109 (1998), 7764-7776

[41] M.K. Sabbe, M. Saeys, M.-F. Reyniers, G.B. Marin, V. Van Speybroeck, M. Waroquier, Group additive values for the gas phase standard enthalpy of formation of hydrocarbons and hydrocarbon radicals, J. Phys. Chem. A, 109 (2005), 7466-7480

[42] N.M. Vandewiele, R. Van de Vijver, K.M. Van Geem, M.-F. Reyniers, G.B. Marin, Symmetry calculation for molecules and transition states, J. Comput. Chem., 36 (2015), 181-192

[43] R. Van de Vijver, N.M. Vandewiele, A.G. Vandeputte, K.M. Van Geem, M.-F. Reyniers, W.H. Green, G.B. Marin, Rule-based ab initio kinetic model for alkyl sulfide pyrolysis, Chem. Eng. J., 278 (2015), 385-393

[44] C.F. Goldsmith, G.R. Magoon, W.H. Green, Database of small molecule thermochemistry for combustion, J. Phys. Chem. A, 116 (2012), 9033-9057

[45] M.K. Sabbe, F. De Vleeschouwer, M.-F. Reyniers, M. Waroquier, G.B. Marin, First principles based group additive values for the gas phase standard entropy and heat capacity of hydrocarbons and hydrocarbon radicals, J. Phys. Chem. A, 112 (2008), 12235-12251

[46] P.D. Paraskevas, M.K. Sabbe, M.-F. Reyniers, N.G. Papayannakos, G.B. Marin, Group additive values for the gas-phase standard enthalpy of formation, entropy and heat capacity of oxygenates, Chem.: Eur. J., 19 (2013), 16431-16452

[47] E. Goos, A. Burcat, B. Ruscic, Extended third millennium thermodynamic database for combustion and air-pollution use with updates from active thermochemical tables, 2014, http://burcat.technion.ac.il/dir/.

[48] S.W. Benson, Thermochemical Kinetics: Methods for the Estimation of Thermochemical Data and Rate Parameters, John Wiley \& Sons, New York (1976)

[49] C.-W. Zhou, Y. Li, E. O'Connor, K.P. Somers, S. Thion, C. Keesee, O. Mathieu, E.L. Petersen, T.A. DeVerter, M.A. Oehlschlaeger, G. Kukkadapu, C.-J. Sung, M. Alrefae, F. Khaled, A. Farooq, P. Dirrenberger, P.-A. Glaude, F. Battin-Leclerc, J. Santner, Y. Ju, T. Held, F.M. Haas, F.L. Dryer, H.J. Curran, A comprehensive experimental and modeling study of isobutene oxidation, Combust. Flame, 167 (2016), 353-379 
[50] S.M. Burke, W. Metcalfe, O. Herbinet, F. Battin-Leclerc, F.M. Haas, J. Santner, F.L. Dryer, H.J. Curran, An experimental and modeling study of propene oxidation. Part 1: Speciation measurements in jet-stirred and flow reactors, Combust. Flame, 161 (2014), 2765-2784

[51] M.M. Kopp, N.S. Donato, E.L. Petersen, W.K. Metcalfe, S.M. Burke, H.J. Curran, Oxidation of ethylene-air mixtures at elevated pressures, part 1: experimental results, J. Propul. Power, 30 (2014), 790-798

[52] W.K. Metcalfe, S.M. Burke, S.S. Ahmed, H.J. Curran, A hierarchical and comparative kinetic modeling study of C1-C2 hydrocarbon and oxygenated fuels, Int. J. Chem. Kinet., 45 (2013), 638675

[53] P. Dagaut, M. Cathonnet, isobutene oxidation and ignition: experimental and detailed kinetic modeling study, Combust. Sci. Technol., 137 (1998), 237-275

[54] P.D. Paraskevas, M.K. Sabbe, M.-F. Reyniers, N.G. Papayannakos, G.B. Marin, Group additive kinetics for hydrogen transfer between oxygenates, J. Phys. Chem. A, 119 (2015), 6961-6980

[55] J. Zádor, S.J. Klippenstein, J.A. Miller, Pressure-dependent OH yields in alkene+HO2 reactions: a theoretical study, J. Phys. Chem. A, 115 (2011), 10218-10225

[56] P.D. Paraskevas, M.K. Sabbe, M.-F. Reyniers, N.G. Papayannakos, G.B. Marin, Kinetic modeling of $\alpha$-hydrogen abstractions from unsaturated and saturated oxygenate compounds by hydrogen atoms, J. Phys. Chem. A, 118 (2014), 9296-9309

[57] M.K. Sabbe, A. Vandeputte, M.-F. Reyniers, M. Waroquier, G.B. Marin, Modeling the influence of resonance stabilization on the kinetics of hydrogen abstractions, Phys. Chem. Chem. Phys., 12 (2010), 1278-1298

[58] M.K. Sabbe, M.-F. Reyniers, M. Waroquier, G.B. Marin, Hydrogen radical additions to unsaturated hydrocarbons and the reverse beta-scission reactions: modeling of activation energies and pre-exponential factors, ChemPhysChem, 11 (2010), 195-210

[59] M.K. Sabbe, M.-F. Reyniers, V. Van Speybroeck, M. Waroquier, G.B. Marin, Carbon-centered radical addition and beta-scission reactions: modeling of activation energies and pre-exponential factors, ChemPhysChem, 9 (2008), 124-140

[60] J. Mendes, C.-W. Zhou, H.J. Curran, Theoretical chemical kinetic study of the H-atom abstraction reactions from aldehydes and acids by $\dot{\mathrm{H}}$ atoms and $\dot{\mathrm{O}} \mathrm{H}, \mathrm{HO} 2$, and $\dot{\mathrm{C}} \mathrm{H} 3$ radicals, J. Phys. Chem. A, 118 (2014), 12089-12104

[61] R. Sivaramakrishnan, J.V. Michael, Rate constants for $\mathrm{OH}$ with selected large alkanes: shocktube measurements and an improved group scheme, J. Phys. Chem. A, 113 (2009), 5047-5060

[62] J. Badra, A. Elwardany, A Farooq, Shock tube measurements of the rate constants for seven large alkanes + OH, Proc. Combust. Inst., 35 (2015), 189-196 
[63] J. Aguilera-Iparraguirre, H.J. Curran, W. Klopper, J.M. Simmie, Accurate benchmark calculation of the reaction barrier height for hydrogen abstraction by the hydroperoxyl radical from methane. implications for $\mathrm{CnH} 2 \mathrm{n}+2$ where $\mathrm{n}=2 \rightarrow 4$, J. Phys. Chem. A, 112 (2008), 7047-7054

[64] G. Mittal, S.M. Burke, V.A. Davies, B. Parajuli, W.K. Metcalfe, H.J. Curran, Autoignition of ethanol in a rapid compression machine, Combust. Flame, 161 (2014), 1164-1171

[65] K. Wang, S.M. Villano, A.M. Dean, Reactivity-structure-based rate estimation rules for alkyl radical $\mathrm{H}$ atom shift and alkenyl radical cycloaddition reactions, J. Phys. Chem. A, 119 (2015), 7205-7221

[66] J. Bugler, K.P. Somers, E.J. Silke, H.J. Curran, Revisiting the kinetics and thermodynamics of the low-temperature oxidation pathways of alkanes: a case study of the three pentane isomers, J. Phys. Chem. A, 119 (2015), 7510-7527

[67] J. Lee, J.W. Bozzelli, Thermochemical and kinetic analysis of the allyl radical with 02 reaction system, Proc. Combust. Inst., 30 (2005), 1015-1022

[68] F. Zhang, T.S. Dibble, Effects of olefin group and its position on the kinetics for intramolecular H-shift and HO2 elimination of alkenyl peroxy radicals, J. Phys. Chem. A, 115 (2011), 655-663

[69] C.K. Westbrook, W.J. Pitz, M. Mehl, P.-A. Glaude, O. Herbinet, S. Bax, F. Battin-Leclerc, O. Mathieu, E.L. Petersen, J. Bugler, H.J. Curran, Experimental and kinetic modeling study of 2-methyl2-butene: allylic hydrocarbon kinetics, J. Phys. Chem. A, 119 (2015), 7462-7480

[70] C.F. Goldsmith, S.J. Klippenstein, W.H. Green, Theoretical rate coefficients for allyl + HO2 and allyloxy decomposition, Proc. Combust. Inst., 33 (2011), 273-282

[71] H. Sun, J.W. Bozzelli, C.K. Law, Thermochemical and kinetic analysis on the reactions of 02 with products from $\mathrm{OH}$ addition to isobutene, 2-hydroxy-1,1-dimethylethyl, and 2-hydroxy-2methylpropyl radicals: HO2 formation from oxidation of neopentane, Part II, J. Phys. Chem. A, 111 (2007), 4974-4986

[72] J. Zádor, R.X. Fernandes, Y. Georgievskii, G. Meloni, C.A. Taatjes, J.A. Miller, The reaction of hydroxyethyl radicals with 02: a theoretical analysis and experimental product study, Proc. Combust. Inst., 32 (2009), 271-277

[73] S.M. Sarathy, S. Vranckx, K. Yasunaga, M. Mehl, P. Oßwald, W.K. Metcalfe, C.K. Westbrook, W.J. Pitz, K. Kohse-Höinghaus, R.X. Fernandes, H.J. Curran, A comprehensive chemical kinetic combustion model for the four butanol isomers, Combust. Flame, 159 (2012), 2028-2055

[74] S.P. Pyl, K.M. Van Geem, P. Puimège, M.K. Sabbe, M.-F. Reyniers, G.B. Marin, A comprehensive study of methyl decanoate pyrolysis, Energy, 43 (2012), 146-160

[75] J.C. Bauge, F. Battin-Leclerc, F. Baronnet, Experimental and modeling study of the oxidation of isobutene, Int. J. Chem. Kinet., 30 (1998), 629-640 
[76] CHEMKIN-PRO, R.J. Kee, F.M. Rupley, J.A. Miller, M.E. Coltrin, J.F. Grcar, E. Meeks, H.K. Moffat, A.E. Lutz, G. Dixon-Lewis, M.D. Smooke, J. Warnatz, G.H. Evans, L.R. S., R.E. Mitchell, L.R. Petzold, W.C. Reynolds, M. Caracotsios, W.E. Stewart, P. Glarborg, C. Wang, O. Adigun, Reaction Design Inc., San Diego, CA, 2010.

[77] K. Brezinsky, F.L. Dryer, A flow reactor study of the oxidation of iso-butylene and an isobutyleneln-octane mixture, Combust. Sci. Technol., 45 (1986), 225-232

[78] C.F. Goldsmith, L.B. Harding, Y. Georgievskii, J.A. Miller, S.J. Klippenstein, Temperature and pressure-dependent rate coefficients for the reaction of vinyl radical with molecular oxygen, J. Phys. Chem. A, 119 (2015), 7766-7779

[79] J. Cai, L. Zhang, F. Zhang, Z. Wang, Z. Cheng, W. Yuan, F. Qi, Experimental and kinetic modeling study of n-butanol pyrolysis and combustion, Energ. Fuel., 26 (2012), 5550-5568

[80] H.J. Curran, P. Gaffuri, W.J. Pitz, C.K. Westbrook, A comprehensive modeling study of nheptane oxidation, Combust. Flame, 114 (1998), 149-177

[81] P.D. Paraskevas, M.K. Sabbe, M.-F. Reyniers, N.G. Papayannakos, G.B. Marin, Kinetic modeling of $\alpha$-hydrogen abstractions from unsaturated and saturated oxygenate compounds by carboncentered radicals, ChemPhysChem, 15 (2014), 1849-1866 